\title{
Research Article \\ Pulsatile Flow of a Two-Fluid Model for Blood Flow through Arterial Stenosis
}

\author{
D. S. Sankar \\ School of Mathematical Sciences, University Science Malaysia, 11800 Penang, Malaysia \\ Correspondence should be addressed to D. S.Sankar, sankar_ds@yahoo.co.in
}

Received 25 January 2010; Accepted 4 April 2010

Academic Editor: Saad A. Ragab

Copyright $(2010$ D. S. Sankar. This is an open access article distributed under the Creative Commons Attribution License, which permits unrestricted use, distribution, and reproduction in any medium, provided the original work is properly cited.

Pulsatile flow of a two-fluid model for blood flow through stenosed narrow arteries is studied through a mathematical analysis. Blood is treated as two-phase fluid model with the suspension of all the erythrocytes in the as Herschel-Bulkley fluid and the plasma in the peripheral layer as a Newtonian fluid. Perturbation method is used to solve the system of nonlinear partial differential equations. The expressions for velocity, wall shear stress, plug core radius, flow rate and resistance to flow are obtained. The variations of these flow quantities with stenosis size, yield stress, axial distance, pulsatility and amplitude are analyzed. It is found that pressure drop, plug core radius, wall shear stress and resistance to flow increase as the yield stress or stenosis size increases while all other parameters held constant. It is observed that the percentage of increase in the magnitudes of the wall shear stress and resistance to flow over the uniform diameter tube is considerably very low for the present two-fluid model compared with that of the single-fluid model of the HerschelBulkley fluid. Thus, the presence of the peripheral layer helps in the functioning of the diseased arterial system.

\section{Introduction}

The analysis of blood flow through stenosed arteries is very important because of the fact that the cause and development of many arterial diseases leading to the malfunction of the cardiovascular system are, to a great extent, related to the flow characteristics of blood together with the geometry of the blood vessels. Among the various arterial diseases, the development of arteriosclerosis in blood vessels is quite common which may be attributed to the accumulation of lipids in the arterial wall or pathological changes in the tissue structure [1]. Arteries are narrowed by the development of atherosclerotic plaques that protrude into the lumen, resulting in stenosed arteries. When an obstruction is developed in an artery, one of the most serious consequences is the increased resistance and the associated reduction of the blood flow to the particular vascular bed supplied by the artery. Also, the continual flow of blood may lead to shearing of the superficial layer of the plaques, parts of which may be 
deposited in some other blood vessel forming thrombus. Thus, the presence of a stenosis can lead to the serious circulatory disorder.

Several theoretical and experimental attempts have been made to study the blood flow characteristics due to the presence of a stenosis in the arterial lumen of a blood vessel [2-10]. It has been reported that the hydrodynamic factors play an important role in the formation of stenosis $[11,12]$ and hence, the study of the blood flow through a stenosed tube is very important. Many authors have dealt with this problem treating blood as a Newtonian fluid and assuming the flow to be steady [13-16]. Since the blood flow through narrow arteries is highly pulsatile, more attempts have been made to study the pulsatile flow of blood treating blood as a Newtonian fluid [3, 6-8, 17-19]. The Newtonian behavior may be true in larger arteries, but, blood, being a suspension of cells in plasma, exhibits nonNewtonian behavior at low-shear rates $(\dot{\gamma}<10 / \mathrm{scc})$ in small diameter arteries $(0.02 \mathrm{~mm}-0.1 \mathrm{~mm})$; particularly, in diseased state, the actual flow is distinctly pulsatile [2, 20-25]. Several attempts have been made to study the nonNewtonian behavior and pulsatile flow of blood through stenosed tubes $[2,4,9,10,26-28]$.

Bugliarello and Sevilla [29] and Cokelet [30] have shown experimentally that for blood flowing through narrow blood vessels, there is an outer phase (peripheral layer) of plasma (Newtonian fluid) and an inner phase (core region) of suspension of all the erythrocytes as a nonNewtonian fluid. Their experimentally measured velocity profiles in the tubes confirm the impossibility of representing the velocity distribution by a single-phase fluid model which ignores the presence of the peripheral layer (outer layer) that plays a crucial role in determining the flow patterns of the system. Thus, for a realistic description of blood flow, perhaps, it is more appropriate to treat blood as a two-phase fluid model consisting of a core region (inner phase) containing all the erythrocytes as a nonNewtonian fluid and a peripheral layer (outer phase) of plasma as a Newtonian fluid. Several researchers have studied the two-phase fluid models for blood flow through stenosed arteries treating the fluid in the inner phase as a nonNewtonian fluid and the fluid in the outer phase as a Newtonian fluid [25, 26, 31-33]. Srivastava and Saxena [25] have analyzed a two-phase fluid model for blood flow through stenosed arteries treating the suspension of all the erythrocytes in the core region (inner phase) as a Casson fluid and the plasma in the peripheral layer (outer phase) is represented by a Newtonian fluid. In the present model, we study a two-phase fluid model for pulsatile flow of blood through stenosed narrow arteries assuming the fluid in the core region as a Herschel-Bulkley fluid while the fluid in the peripheral region is represented by a Newtonian fluid.

Chaturani and Ponnalagar Samy [28] and Sankar and Hemalatha [2] have mentioned that for tube diameter $0.095 \mathrm{~mm}$ blood behaves like Herschel-Bulkley fluid rather than power law and Bingham fluids. Iida [34] reports "The velocity profile in the arterioles having diameter less than $0.1 \mathrm{~mm}$ are generally explained fairly by the Casson and Herschel-Bulkley fluid models. However, the velocity profile in the arterioles whose diameters less than $0.0650 \mathrm{~mm}$ does not conform to the Casson fluid model, but, can still be explained by the Herschel-Bulkley model". Furthermore, the Herschel-Bulkley fluid model can be reduced to the Newtonian fluid model, power law fluid model and Bingham fluid model for appropriate values of the power law index $(n)$ and yield index $\left(\bar{\tau}_{y}\right)$. Since the Herschel-Bulkley fluid model's constitutive equation has one more parameter than the Casson fluid model; one can get more detailed information about the flow characteristics by using the Herschel-Bulkley fluid model. Moreover, the Herschel-Bulkley fluid model could also be used to study the blood flow through larger arteries, since the Newtonian fluid model can be obtained as a particular case of this model. Hence, we felt that it is appropriate to represent the fluid in 


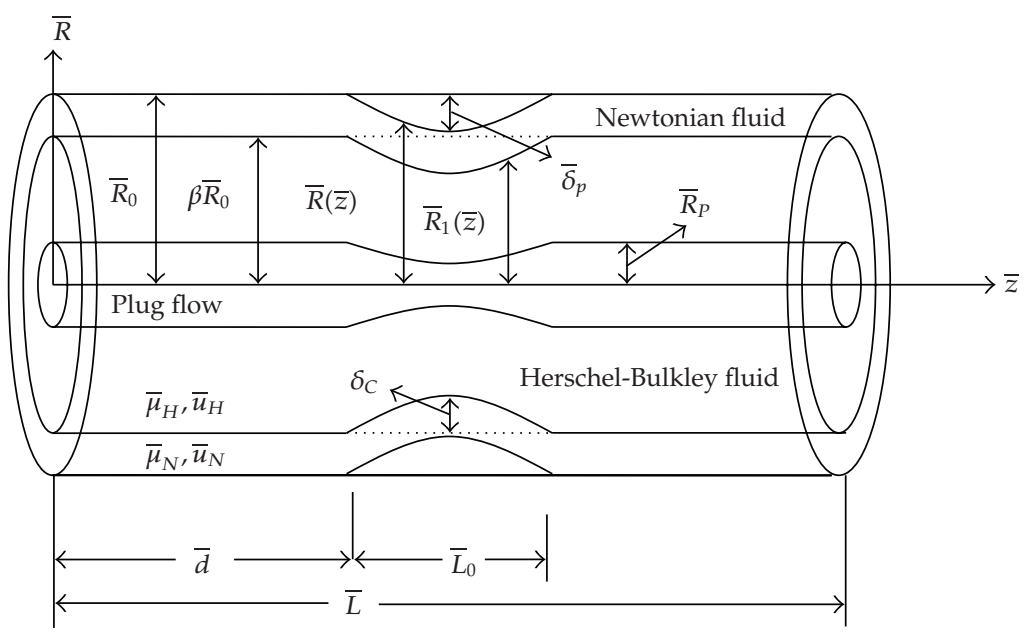

Figure 1: Flow geometry of an arterial stenosis with peripheral layer.

the core region of the two-phase fluid model by the Herschel-Bulkley fluid model rather than the Casson fluid model. Thus, in this paper, we study a two-phase fluid model for blood flow through mild stenosed narrow arteries (of diameter $0.02 \mathrm{~mm}-0.1 \mathrm{~mm}$ ) at low-shear rates $(\dot{\gamma}<10 / \mathrm{sec})$ treating the fluid in the core region (inner phase) as a Herschel-Bulkley fluid and the plasma in the peripheral region (outer phase) as a Newtonian fluid.

In this study, the effects of the pulsatility, stenosis, peripheral layer and the nonNewtonian behavior of blood are analyzed using an analytical solution. Section 2 formulates the problem mathematically and then nondimensionalises the governing equations and boundary conditions. In Section 3, the resulting nonlinear coupled implicit system of differential equations is solved using the perturbation method. The expressions for the velocity, flow rate, wall shear stress, plug core radius, and resistance to flow have been obtained. Section 4 analyses the variations of these flow quantities with stenosis height, yield stress, amplitude, power law index and pulsatile Reynolds number through graphs. The estimates of wall shear stress increase factor and the increase in resistance to flow factor are calculated for the two-phase Herschel-bulkley fluid model and single-phase fluid model.

\section{Mathematical Formulation}

Consider an axially symmetric, laminar, pulsatile and fully developed flow of blood (assumed to be incompressible) in the $\bar{z}$ direction through a circular artery with an axially symmetric mild stenosis. It is assumed that the walls of the artery are rigid and the blood is represented by a two-phase fluid model with an inner phase (core region) of suspension of all erythrocytes as a Herschel-Bulkley fluid and an outer phase (peripheral layer) of plasma as a Newtonian fluid. The geometry of the stenosis is shown in Figure 1. We have used the cylindrical polar coordinates $(\bar{r}, \bar{\phi}, \bar{z})$ whose origin is located on the vessel (stenosed artery) axis. It can be shown that the radial velocity is negligibly small and can be neglected for a low Reynolds number flow in a tube with mild stenosis. In this case, the basic momentum equations governing the flow are

$$
\bar{\rho}_{H} \frac{\partial \bar{u}_{H}}{\partial \bar{t}}=-\frac{\partial \bar{p}}{\partial \bar{z}}-\frac{1}{\bar{r}} \frac{\partial}{\partial \bar{r}}\left(\bar{r} \bar{\tau}_{H}\right) \quad \text { in } 0 \leq \bar{r} \leq \bar{R}_{1}(\bar{z}),
$$




$$
\begin{gathered}
\bar{\rho}_{N} \frac{\partial \bar{u}_{N}}{\partial \bar{t}}=-\frac{\partial \bar{p}}{\partial \bar{z}}-\frac{1}{\bar{r}} \frac{\partial}{\partial \bar{r}}\left(\bar{r} \bar{\tau}_{N}\right) \quad \text { in } \bar{R}_{1}(\bar{z}) \leq \bar{r} \leq \bar{R}(\bar{z}) \\
0=-\frac{\partial \bar{p}}{\partial \bar{r}^{\prime}}
\end{gathered}
$$

where the shear stress $\bar{\tau}=\left|\bar{\tau}_{\bar{r}}\right|=-\bar{\tau}_{\bar{r} \bar{z}}$ (since $\bar{\tau}=\bar{\tau}_{H}$ or $\bar{\tau}=\bar{\tau}_{N}$ ). Herschel-Bulkley fluid is a nonNewtonian fluid which is widely used in many areas of fluid dynamics, for example, dam break flows, flow of polymers, blood, and semisolids. Herschel-Bulkley fluid is a nonNewtonian fluid with nonzero yield stress which is generally used in the studies of blood flow through narrow arteries at low-shear rate. Herschel-Bulkley equation is an empirical relation which connects shear stress and shear rate through the viscosity which is given in (2.4) and (2.5). The relations between the shear stress and the strain rate of the fluids in motion in the core region (for Herschel-Bulkley fluid) and in the peripheral region (for Newtonian fluid) are given by

$$
\begin{gathered}
\bar{\tau}_{H}=\sqrt[n]{\bar{\mu}_{H}\left(\frac{\partial \bar{u}_{H}}{\partial \bar{r}}\right)}+\bar{\tau}_{y} \quad \text { if } \bar{\tau}_{H} \geq \bar{\tau}_{y}, \bar{R}_{p} \leq \bar{r} \leq \bar{R}_{1}(\bar{z}) \\
\frac{\partial \bar{u}_{H}}{\partial \bar{r}}=0 \quad \text { if } \bar{\tau}_{H} \leq \bar{\tau}_{y}, 0 \leq \bar{r} \leq \bar{R}_{p} \\
\bar{\tau}_{N}=\bar{\mu}_{N}\left(-\frac{\partial \bar{u}_{N}}{\partial \bar{r}}\right) \quad \text { if } \bar{R}_{1}(\bar{z}) \leq \bar{r} \leq \bar{R}(\bar{z})
\end{gathered}
$$

where $\bar{u}_{H}, \bar{u}_{N}$ are the axial component of the fluid's velocity in the core region and peripheral region; $\bar{\tau}_{H}, \bar{\tau}_{N}$ are the shear stress of the Herschel-Bulkley fluid and Newtonian fluid; $\bar{\mu}_{H}, \bar{\mu}_{N}$ are the viscosities of the Herschel-Bulkley fluid and Newtonian fluid with respective dimensions $\left[M L^{-1} T^{-2}\right]^{n} T$ and $M L^{-1} T^{-1} ; \bar{\rho}_{H}, \bar{\rho}_{N}$ are the densities of the Herschel-Bulkley fluid and Newtonian fluid; $\bar{p}$ is the pressure, $\bar{t}$; is the time; $\bar{\tau}_{y}$ is the yield stress. From (2.5), it is clear that the velocity gradient vanishes in the region where the shear stress is less than the yield stress which implies a plug flow whenever $\bar{\tau}_{H} \leq \bar{\tau}_{y}$. However, the fluid behavior is indicated whenever $\bar{\tau}_{H} \geq \bar{\tau}_{y}$. The geometry of the stenosis in the peripheral region as shown in Figure 1 is given by

$$
\bar{R}(\bar{z})= \begin{cases}\bar{R}_{0} & \text { in the normal artery region, } \\ \bar{R}_{0}-\frac{\bar{\delta}_{p}}{2}\left[1+\cos \frac{2 \pi}{\bar{L}_{0}}\left(\bar{z}-\bar{d}-\frac{\bar{L}_{0}}{2}\right)\right] & \text { in } \bar{d} \leq \bar{z} \leq \bar{d}+\bar{L}_{0}\end{cases}
$$

where $\bar{R}(\bar{z})$ is the radius of the stenosed artery with peripheral layer, $\bar{R}_{0}$ is the radius of the normal artery, $\bar{L}_{0}$ is the length of the stenosis, $\bar{d}$ indicates its location, and $\bar{\delta}_{p}$ is the maximum depth of the stenosis in the peripheral layer such that $\left[\bar{\delta}_{P} / \bar{R}_{0}\right] \ll 1$. The geometry of the 
stenosis in the core region as seen in Figure 1 is given by

$$
\bar{R}_{1}(\bar{z})= \begin{cases}\beta \bar{R}_{0} & \text { in the normal artery region, } \\ \beta \bar{R}_{0}-\frac{\bar{\delta}_{C}}{2}\left[1+\cos \frac{2 \pi}{\bar{L}_{0}}\left(\bar{z}-\bar{d}-\frac{\bar{L}_{0}}{2}\right)\right] & \text { in } \bar{d} \leq \bar{z} \leq \bar{d}+\bar{L}_{0},\end{cases}
$$

where $\bar{R}_{1}(\bar{z})$ is the radius of the stenosed core region of the artery, $\beta$ is the ratio of the central core radius to the normal artery radius, $\beta \bar{R}_{0}$ is the radius of the core region of the normal artery, and $\bar{\delta}_{C}$ is the maximum depth of the stenosis in the core region such that $\left[\bar{\delta}_{C} / \bar{R}_{0}\right] \ll 1$. The boundary conditions are

$$
\begin{aligned}
& \text { (i) } \bar{\tau}_{H} \quad \text { is finite and } \frac{\partial \bar{u}_{H}}{\partial \bar{r}}=0 \quad \text { at } \bar{r}=0, \\
& \text { (ii) } \bar{\tau}_{H}=\bar{\tau}_{N} \quad \text { at } \bar{r}=\bar{R}_{1}(\bar{z}), \\
& \text { (iii) } \bar{u}_{H}=\bar{u}_{N} \quad \text { at } \bar{r}=\bar{R}_{1}(\bar{z}), \\
& \text { (iv) } \bar{u}_{N}=0 \quad \text { at } \bar{r}=\bar{R}(\bar{z}) .
\end{aligned}
$$

Since the pressure gradient is a function of $\bar{z}$ and $\bar{t}$, we take

$$
-\frac{\partial \bar{p}}{\partial \bar{z}}=\bar{q}(\bar{z}) f(\bar{t})
$$

where $\bar{q}(\bar{z})=-(\partial \bar{p} / \partial \bar{z})(\bar{z}, 0), f(\bar{t})=1+A \sin \bar{\omega} \bar{t}, A$ is the amplitude of the flow and $\bar{\omega}$ is the angular frequency of the blood flow. Since any periodic function can be expanded in a series of sines of multiple angles using Fourier series, it is reasonable to choose $f(\bar{t})=1+A \sin \bar{\omega} \bar{t}$ as a good approximation. We introduce the following nondimensional variables

$$
\begin{gathered}
z=\frac{\bar{z}}{\bar{R}_{0}}, \quad R(z)=\frac{\bar{R}(\bar{z})}{\bar{R}_{0}}, \quad R_{1}(z)=\frac{\bar{R}_{1}(\bar{z})}{\bar{R}_{0}}, \quad r=\frac{\bar{r}}{\bar{R}_{0}}, \quad t=\bar{\omega} \bar{t}, \quad d=\frac{\bar{d}}{\bar{R}_{0}}, \quad L_{0}=\frac{\bar{L}_{0}}{\bar{R}_{0}}, \\
q(z)=\frac{\bar{q}(\bar{z})}{\bar{q}_{0}}, \quad u_{H}=\frac{\bar{u}_{H}}{\bar{q}_{0} \bar{R}_{0}^{2} / 4 \bar{\mu}_{0}}, \quad u_{N}=\frac{\bar{u}_{N}}{\bar{q}_{0} \bar{R}_{0}^{2} / 4 \bar{\mu}_{N}}, \quad \tau_{H}=\frac{\bar{\tau}_{H}}{\bar{q}_{0} \bar{R}_{0} / 2}, \quad \tau_{N}=\frac{\bar{\tau}_{N}}{\bar{q}_{0} \bar{R}_{0} / 2}, \\
\theta=\frac{\bar{\tau}_{y}}{\bar{q}_{0} \bar{R}_{0} / 2}, \quad \alpha_{H}^{2}=\frac{\bar{R}_{0}^{2} \bar{\omega} \bar{\rho}_{H}}{\bar{\mu}_{0}}, \quad \alpha_{N}^{2}=\frac{\bar{R}_{0}^{2} \bar{\omega} \bar{\rho}_{N}}{\bar{\mu}_{N}}, \quad R_{p}=\frac{\bar{R}_{p}}{\bar{R}_{0}}, \quad \delta_{p}=\frac{\bar{\delta}_{p}}{\bar{R}_{0}}, \quad \delta_{C}=\frac{\bar{\delta}_{C}}{\bar{R}_{0}},
\end{gathered}
$$

where $\bar{\mu}_{0}=\bar{\mu}_{H}\left(2 / \bar{q}_{0} \bar{R}_{0}\right)^{n-1}$, having the dimension as that of the Newtonian fluid's viscosity, $\bar{q}_{0}$ is the negative of the pressure gradient in the normal artery, $\alpha_{H}$ is the pulsatile Reynolds number or generalized Wormersly frequency parameter and when $n=1$, we get 
the Wormersly frequency parameter $\alpha_{N}$ of the Newtonian fluid. Using the nondimensional variables, (2.1), (2.2), (2.4), (2.5), and (2.6) reduce, respectively, to

$$
\begin{gathered}
\alpha_{H}^{2} \frac{\partial u_{H}}{\partial t}=4 q(z) f(t)-\frac{2}{r} \frac{\partial}{\partial r}\left(r \tau_{H}\right) \quad \text { if } 0 \leq r \leq R_{1}(z) \\
\alpha_{N}^{2} \frac{\partial u_{N}}{\partial t}=4 q(z) f(t)-\frac{2}{r} \frac{\partial}{\partial r}\left(r \tau_{N}\right) \quad \text { if } R_{1}(z) \leq r \leq R(z), \\
\tau_{H}=\sqrt[n]{-\frac{1}{2} \frac{\partial u_{H}}{\partial r}}+\theta \quad \text { if } \tau_{H} \geq \theta, R_{p} \leq r \leq R_{1}(z) \\
\frac{\partial u_{H}}{\partial r}=0 \quad \text { if } \tau_{H} \leq \theta, 0 \leq r \leq R_{p} \\
\tau_{N}=-\frac{1}{2} \frac{\partial u_{N}}{\partial r} \quad \text { if } R_{1}(z) \leq r \leq R(z)
\end{gathered}
$$

where $f(t)=1+A \sin t$. The boundary conditions (in dimensionless form) are

$$
\begin{aligned}
& \text { (i) } \tau_{H} \quad \text { is finite at } r=0, \\
& \text { (ii) } \frac{\partial u_{H}}{\partial r}=0 \quad \text { at } r=0, \\
& \text { (iii) } \tau_{H}=\tau_{N} \quad \text { at } r=R_{1}(z), \\
& \text { (iv) } u_{H}=u_{N} \text { at } r=R_{1}(z), \\
& \text { (v) } u_{N}=0 \text { at } r=R(z) .
\end{aligned}
$$

The geometry of the stenosis in the peripheral region (in dimensionless form) is given by

$$
R(z)= \begin{cases}1 & \text { in the normal artery region, } \\ 1-\frac{\delta_{p}}{2}\left[1+\cos \frac{2 \pi}{L_{0}}\left(z-d-\frac{L_{0}}{2}\right)\right] & \text { in } d \leq z \leq d+L_{0} .\end{cases}
$$

The geometry of the stenosis in the core region (in dimensionless form) is given by

$$
R_{1}(z)= \begin{cases}\beta & \text { in the normal artery region, } \\ \beta-\frac{\delta_{C}}{2}\left[1+\cos \frac{2 \pi}{L_{0}}\left(z-d-\frac{L_{0}}{2}\right)\right] & \text { in } d \leq z \leq d+L_{0} .\end{cases}
$$

The nondimensional volume flow rate $Q$ is given by

$$
Q=4 \int_{0}^{R(z)} u(r, z, t) r d r
$$

where $Q=\bar{Q} /\left[\pi \bar{R}_{0}^{4} \bar{q}_{0} / 8 \bar{\mu}_{0}\right], \bar{Q}$ is the volume flow rate. 


\section{Method of Solution}

When we nondimensionalize the constitutive equations (2.1) and (2.2), $\alpha_{H}^{2}$ and $\alpha_{N}^{2}$ occur naturally and these pulsatile Reynolds numbers are time dependent and hence, it is more appropriate to expand (2.12)-(2.16) about $\alpha_{H}^{2}$ and $\alpha_{N}^{2}$. The plug core velocity $u_{p}$, the velocity in the core region $u_{H}$, the velocity in the peripheral region $u_{N}$, the plug core shear stress $\tau_{p}$, the shear stress in the core region $\tau_{H}$, the shear stress in the peripheral region $\tau_{N}$, and the plug core radius $R_{p}$ are expanded as follows in terms of $\alpha_{H}^{2}$ and $\alpha_{N}^{2}$ (where $\alpha_{H}^{2} \ll 1$ and $\left.\alpha_{N}^{2} \ll 1\right)$ :

$$
\begin{gathered}
u_{P}(z, t)=u_{0 P}(z, t)+\alpha_{H}^{2} u_{1 P}(z, t)+\cdots, \\
u_{H}(r, z, t)=u_{0 H}(r, z, t)+\alpha_{H}^{2} u_{1 H}(r, z, t)+\cdots, \\
u_{N}(r, z, t)=u_{0 N}(r, z, t)+\alpha_{N}^{2} u_{1 N}(r, z, t)+\cdots, \\
\tau_{P}(z, t)=\tau_{0 P}(z, t)+\alpha_{H}^{2} \tau_{1 P}(z, t)+\cdots, \\
\tau_{H}(r, z, t)=\tau_{0 H}(r, z, t)+\alpha_{H}^{2} \tau_{1 H}(r, z, t)+\cdots, \\
\tau_{N}(r, z, t)=\tau_{0 N}(r, z, t)+\alpha_{N}^{2} \tau_{1 N}(r, z, t)+\cdots, \\
R_{P}(z, t)=R_{0 P}(z, t)+\alpha_{H}^{2} R_{1 P}(z, t)+\cdots
\end{gathered}
$$

Substituting (3.2), (3.5) in (2.12) and then equating the constant terms and $\alpha_{H}^{2}$ terms, we obtain

$$
\begin{aligned}
& \frac{\partial}{\partial r}\left(r \tau_{0 H}\right)=2 q(z) f(t) r, \\
& \frac{\partial u_{0 H}}{\partial t}=-\frac{2}{r} \frac{\partial}{\partial r}\left(r \tau_{1 H}\right) .
\end{aligned}
$$

Applying (3.2), (3.5) in (2.14) and then equating the constant terms and $\alpha_{H}^{2}$ terms, one can get

$$
\begin{gathered}
-\frac{\partial u_{0 H}}{\partial r}=2 \tau_{0 H}^{n-1}\left[\tau_{0 H}-n \theta\right] \\
-\frac{\partial u_{1 H}}{\partial r}=2 n \tau_{0 H}^{n-2} \tau_{1 H}\left[\tau_{0 H}-(n-1) \theta\right] .
\end{gathered}
$$

Using (3.3) and (3.6) in (2.13) and then equating the constant terms and $\alpha_{N}^{2}$ terms, we get

$$
\begin{aligned}
& \frac{\partial}{\partial r}\left(r \tau_{0 N}\right)=2 q(z) f(t) r, \\
& \frac{\partial u_{0 N}}{\partial t}=-\frac{2}{r} \frac{\partial}{\partial r}\left(r \tau_{1 N}\right) .
\end{aligned}
$$


On substituting (3.3) and (3.6) in (2.16) and then equating the constant terms and $\alpha_{N}^{2}$ terms, one can obtain

$$
\begin{aligned}
-\frac{\partial u_{0 N}}{\partial r} & =2 \tau_{0 N}, \\
-\frac{\partial u_{1 N}}{\partial r} & =2 \tau_{1 N} .
\end{aligned}
$$

Using (3.1)-(3.6) in (2.17) and then equating the constant terms and $\alpha_{H}^{2}$ and $\alpha_{N}^{2}$ terms, the boundary conditions are simplified, respectively, to

$$
\begin{gathered}
\tau_{0 P}, \tau_{1 P} \quad \text { are finite at } r=0, \\
\frac{\partial u_{0 P}}{\partial r}=0, \quad \frac{\partial u_{1 P}}{\partial r}=0 \quad \text { at } r=0, \\
\tau_{0 H}=\tau_{0 N} \quad \text { at } r=R_{1}(z), \\
\tau_{1 H}=\tau_{1 N} \quad \text { at } r=R_{1}(z), \\
u_{0 H}=u_{0 N} \quad \text { at } r=R_{1}(z), \\
u_{1 H}=u_{1 N} \quad \text { at } r=R_{1}(z), \\
u_{0 N}=0 \quad \text { at } r=R(z), \\
u_{1 N}=0 \quad \text { at } r=R(z) .
\end{gathered}
$$

Equations (3.8)-(3.11) and (3.12)-(3.15) are the system of differential equations which can be solved for the unknowns $u_{0 H}, u_{1 H}, \tau_{0 H}, \tau_{1 H}$ and $u_{0 N}, u_{1 N}, \tau_{0 N}, \tau_{1 N}$, respectively, with the help of boundary conditions (3.16)-(3.23). Integrating (3.8) between 0 and $R_{0 P}$ and applying the boundary condition (3.16), we get

$$
\tau_{0 P}=q(z) f(t) R_{0 P} .
$$

Integrating (3.8) between $R_{0 P}$ and $r$ and then making use of (3.24), we get

$$
\tau_{0 H}=q(z) f(t) r
$$

Integrating (3.12) between $R_{1}$ and $r$ and then using (3.18), one can get

$$
\tau_{0 N}=q(z) f(t) r
$$

Integrating (3.14) between $r$ and $R$ and then making use of (3.22), we obtain

$$
u_{0 N}=q(z) f(t) R^{2}\left[1-\left(\frac{r}{R}\right)^{2}\right]
$$


Integrating (3.10) between $r$ and $R_{1}$ and using the boundary condition (3.20), we get

$$
\begin{aligned}
u_{0 H}= & {[q(z) f(t) R] R\left\{1-\left(\frac{R_{1}}{R}\right)^{2}\right\} } \\
& +2\left[q(z) f(t) R_{1}\right]^{n} R_{1}\left[\frac{1}{(n+1)}\left\{1-\left(\frac{r}{R_{1}}\right)^{n+1}\right\}-\frac{k^{2}}{R_{1}}\left\{1-\left(\frac{r}{R_{1}}\right)^{n}\right\}\right],
\end{aligned}
$$

where $k^{2}=\theta /[q(z) f(t)]$. The plug core velocity $u_{0 P}$ can be obtained from (3.28) by replacing $r$ by $R_{0 P}$ as

$$
\begin{aligned}
u_{0 P}= & {[q(z) f(t) R] R\left\{1-\left(\frac{R_{1}}{R}\right)^{2}\right\} } \\
& +2\left[q(z) f(t) R_{1}\right]^{n} R_{1}\left[\frac{1}{(n+1)}\left\{1-\left(\frac{R_{0 p}}{R_{1}}\right)^{n+1}\right\}-\frac{k^{2}}{R_{1}}\left\{1-\left(\frac{R_{0 p}}{R_{1}}\right)^{n}\right\}\right] .
\end{aligned}
$$

Neglecting the terms with $\alpha_{H}^{2}$ and higher powers of $\alpha_{H}$ in (3.7) and using (3.24), the expression for $R_{0 P}$ is obtained as

$$
\left.r\right|_{\tau_{0 P}=\theta}=R_{0 P}=\left(\frac{\theta}{q(z) f(t)}\right)=k^{2}
$$

Similarly, solving (3.9), (3.11), (3.13), and (3.15) with the help of (3.24)-(3.29), and using (3.19), (3.21) and (3.23), the expressions for $\tau_{1 P}, \tau_{1 H}, \tau_{1 N}, u_{1 H}$, and $u_{1 P}$ can be obtained as

$$
\begin{aligned}
\tau_{1 P}= & -\frac{1}{4}[q(z) f(t) R] B R^{2}\left(\frac{k^{2}}{R}\right)\left\{1-\left(\frac{R_{1}}{R}\right)^{2}\right\} \\
& -\left[q(z) f(t) R_{1}\right]^{n} B R_{1}^{2}\left[\frac{n}{2(n+1)}\left(\frac{k^{2}}{R_{1}}\right)-\frac{(n-1)}{2}\left(\frac{k^{2}}{R_{1}}\right)^{2}-\frac{n}{2(n+1)}\left(\frac{k^{2}}{R_{1}}\right)^{n+2}\right],
\end{aligned}
$$




$$
\begin{aligned}
& \tau_{1 H}=-\frac{1}{4}[q(z) f(t) R] B R^{2}\left(\frac{r}{R}\right)\left\{1-\left(\frac{R_{1}}{R}\right)^{2}\right\}-\left[q(z) f(t) R_{1}\right]^{n} B R_{1}^{2} \\
& \times\left[\frac{n}{(n+1)(n+3)}\left\{\left(\frac{n+3}{2}\right)\left(\frac{r}{R_{1}}\right)-\left(\frac{r}{R_{1}}\right)^{n+2}\right\}\right. \\
& -\frac{(n-1)}{(n+2)}\left(\frac{k^{2}}{R_{1}}\right)\left\{\left(\frac{n+2}{2}\right)\left(\frac{r}{R_{1}}\right)-\left(\frac{r}{R_{1}}\right)^{n+1}\right\} \\
& \left.-\frac{3\left(n^{2}+2 n-2\right)}{2(n+2)(n+3)}\left(\frac{k^{2}}{R_{1}}\right)^{n+3}\left(\frac{R_{1}}{r}\right)\right] \text {, } \\
& \tau_{1 N}=-[q(z) f(t) R] B R R_{1}\left[\frac{1}{4}\left(\frac{r}{R_{1}}\right)-\frac{1}{8}\left(\frac{R_{1}}{R}\right)^{2}\left(\frac{R_{1}}{r}\right)-\frac{1}{8}\left(\frac{R_{1}}{R}\right)^{2}\left(\frac{r}{R_{1}}\right)^{3}\right] \\
& -\left[q(z) f(t) R_{1}\right]^{n} B R_{1}^{2}\left[\frac{n}{2(n+3)}\left(\frac{R_{1}}{r}\right)-\frac{n(n-1)}{2(n+2)}\left(\frac{k^{2}}{R_{1}}\right)\left(\frac{R_{1}}{r}\right)\right. \\
& \left.-\frac{3\left(n^{2}+2 n-2\right)}{2(n+2)(n+3)}\left(\frac{k^{2}}{R_{1}}\right)^{n+3}\left(\frac{R_{1}}{r}\right)\right] \text {, } \\
& u_{1 N}=-2[q(z) f(t) R] B R^{2} R_{1}\left[\frac{1}{8}\left(\frac{R}{R_{1}}\right)\left\{1-\left(\frac{r}{R}\right)^{2}\right\}\right. \\
& \left.-\frac{1}{8}\left(\frac{R_{1}}{R}\right)^{3} \log \left(\frac{R}{r}\right)-\frac{1}{32}\left(\frac{R}{R_{1}}\right)\left\{1-\left(\frac{r}{R}\right)^{4}\right\}\right] \\
& -2\left[q(z) f(t) R_{1}\right]^{n} B R_{1}^{3} \log \left(\frac{R}{r}\right)\left[\frac{n}{2(n+3)}-\frac{n(n-1)}{2(n+2)}\left(\frac{k^{2}}{R_{1}}\right)\right. \\
& \left.-\frac{3\left(n^{2}+2 n-2\right)}{2(n+2)(n+3)}\left(\frac{k^{2}}{R_{1}}\right)^{n+3}\right] \\
& u_{1 H}=-2[q(z) f(t) R] B R^{2} R_{1}\left[\frac{3}{32}\left(\frac{R}{R_{1}}\right)-\frac{1}{8}\left(\frac{R_{1}}{R}\right)+\frac{1}{32}\left(\frac{R_{1}}{R}\right)^{3}+\frac{1}{8}\left(\frac{R_{1}}{R}\right)^{3} \log \left(\frac{R_{1}}{R}\right)\right] \\
& +2\left[q(z) f(t) R_{1}\right]^{n} B R_{1}^{3} \log \left(\frac{R_{1}}{R}\right) \\
& \times\left[\frac{n}{2(n+3)}-\frac{n(n-1)}{2(n+2)}\left(\frac{k^{2}}{R_{1}}\right)-\frac{3\left(n^{2}+2 n-2\right)}{2(n+2)(n+3)}\left(\frac{k^{2}}{R_{1}}\right)^{n+3}\right] \\
& -n\left[q(z) f(t) R_{1}\right]^{n} B R_{1} R^{2}\left\{1-\left(\frac{R_{1}}{R}\right)^{2}\right\}
\end{aligned}
$$




$$
\begin{aligned}
& \times\left[\frac{1}{2(n+1)}\left\{1-\left(\frac{r}{R_{1}}\right)^{n+1}\right\}-\frac{(n-1)}{2 n}\left(\frac{k^{2}}{R_{1}}\right)\left\{1-\left(\frac{r}{R_{1}}\right)^{n}\right\}\right]-2 n\left[q(z) f(t) R_{1}\right]^{2 n-1} B R_{1}^{3} \\
& \times\left[\frac{n}{2(n+1)^{2}}\left\{1-\left(\frac{r}{R_{1}}\right)^{n+1}\right\}-\frac{(n-1)}{2(n+1)}\left(\frac{k^{2}}{R_{1}}\right)\left\{1-\left(\frac{r}{R_{1}}\right)^{n}\right\}\right. \\
& -\frac{n}{2(n+1)^{2}(n+3)}\left\{1-\left(\frac{r}{R_{1}}\right)^{2 n+2}\right\} \\
& +\frac{(n-1)\left(2 n^{2}+6 n+3\right)}{(n+1)(n+2)(n+3)(2 n+1)}\left(\frac{k^{2}}{R_{1}}\right)\left\{1-\left(\frac{r}{R_{1}}\right)^{2 n+1}\right\} \\
& -\frac{(n-1)}{2(n+1)}\left(\frac{k^{2}}{R_{1}}\right)\left\{1-\left(\frac{r}{R_{1}}\right)^{n+1}\right\}+\frac{(n-1)^{2}}{2 n}\left(\frac{k^{2}}{R_{1}}\right)^{2}\left\{1-\left(\frac{r}{R_{1}}\right)^{n}\right\} \\
& -\frac{(n-1)^{2}}{2 n(n+2)}\left(\frac{k^{2}}{R_{1}}\right)^{2}\left\{1-\left(\frac{r}{R_{1}}\right)^{2 n}\right\} \\
& -\frac{3\left(n^{2}+2 n-2\right)}{2(n-1)(n+2)(n+3)}\left(\frac{k^{2}}{R_{1}}\right)^{n+3}\left\{1-\left(\frac{r}{R_{1}}\right)^{n-1}\right\} \\
& \left.+\frac{3(n-1)\left(n^{2}+2 n-2\right)}{2(n-2)(n+2)(n+3)}\left(\frac{k^{2}}{R_{1}}\right)^{n+4}\left\{1-\left(\frac{r}{R_{1}}\right)^{n-2}\right\}\right] \text {, } \\
& u_{1 P}=-2[q(z) f(t) R] B R^{2} R_{1}\left[\frac{3}{32}\left(\frac{R}{R_{1}}\right)-\frac{1}{8}\left(\frac{R_{1}}{R}\right)+\frac{1}{32}\left(\frac{R_{1}}{R}\right)^{3}+\frac{1}{8}\left(\frac{R_{1}}{R}\right)^{3} \log \left(\frac{R_{1}}{R}\right)\right] \\
& +2\left[q(z) f(t) R_{1}\right]^{n} B R_{1}^{3} \log \left(\frac{R_{1}}{R}\right) \\
& \times\left[\frac{n}{2(n+3)}-\frac{n(n-1)}{2(n+2)}\left(\frac{k^{2}}{R_{1}}\right)-\frac{3\left(n^{2}+2 n-2\right)}{2(n+2)(n+3)}\left(\frac{k^{2}}{R_{1}}\right)^{n+3}\right] \\
& -n\left[q(z) f(t) R_{1}\right]^{n} B R_{1} R^{2}\left\{1-\left(\frac{R_{1}}{R}\right)^{2}\right\} \\
& \times\left[\frac{1}{2(n+1)}\left\{1-\left(\frac{k^{2}}{R_{1}}\right)^{n+1}\right\}-\frac{(n-1)}{2 n}\left(\frac{k^{2}}{R_{1}}\right)\left\{1-\left(\frac{k^{2}}{R_{1}}\right)^{n}\right\}\right]-2 n\left[q(z) f(t) R_{1}\right]^{2 n-1} B R_{1}^{3} \\
& \times\left[\frac{n}{2(n+1)^{2}}\left\{1-\left(\frac{k^{2}}{R_{1}}\right)^{n+1}\right\}-\frac{(n-1)}{2(n+1)}\left(\frac{k^{2}}{R_{1}}\right)\left\{1-\left(\frac{k^{2}}{R_{1}}\right)^{n}\right\}\right.
\end{aligned}
$$




$$
\begin{aligned}
& -\frac{n}{2(n+1)^{2}(n+3)}\left\{1-\left(\frac{k^{2}}{R_{1}}\right)^{2 n+2}\right\} \\
& +\frac{(n-1)\left(2 n^{2}+6 n+3\right)}{(n+1)(n+2)(n+3)(2 n+1)}\left(\frac{k^{2}}{R_{1}}\right)\left\{1-\left(\frac{k^{2}}{R_{1}}\right)^{2 n+1}\right\} \\
& -\frac{(n-1)}{2(n+1)}\left(\frac{k^{2}}{R_{1}}\right)\left\{1-\left(\frac{k^{2}}{R_{1}}\right)^{n+1}\right\}+\frac{(n-1)^{2}}{2 n}\left(\frac{k^{2}}{R_{1}}\right)^{2}\left\{1-\left(\frac{k^{2}}{R_{1}}\right)^{n}\right\} \\
& -\frac{(n-1)^{2}}{2 n(n+2)}\left(\frac{k^{2}}{R_{1}}\right)^{2}\left\{1-\left(\frac{k^{2}}{R_{1}}\right)^{2 n}\right\} \\
& -\frac{3\left(n^{2}+2 n-2\right)}{2(n-1)(n+2)(n+3)}\left(\frac{k^{2}}{R_{1}}\right)^{n+3}\left\{1-\left(\frac{k^{2}}{R_{1}}\right)^{n-1}\right\} \\
& +\frac{3(n-1)\left(n^{2}+2 n-2\right)}{2(n-2)(n+2)(n+3)}\left(\frac{k^{2}}{R_{1}}\right)^{n+4}\left\{1-\left(\frac{k^{2}}{R_{1}}\right)^{n-2}\right\},
\end{aligned}
$$

where $B=[1 / f(t)](d f(t) / d t)$. The expression for velocity $u_{H}$ can be easily obtained from (3.2), (3.28) and (3.35). Similarly, the expressions for $u_{N}, \tau_{H}$, and $\tau_{N}$ can be obtained. The expression for wall shear stress $\tau_{w}$ can be obtained by evaluating $\tau_{N}$ at $r=R$ and is given below:

$$
\begin{aligned}
\tau_{w}=\left(\tau_{0 N}+\alpha_{N}^{2} \tau_{1 N}\right)_{r=R}=\tau_{0 w}+\alpha_{N}^{2} \tau_{1 w} \\
=[q(z) f(t) R]+\alpha_{N}^{2}\left\{-\frac{1}{8}[q(z) f(t) R] B R^{2}\left[1-\left(\frac{R_{1}}{R}\right)^{4}\right]\right\} \\
+\alpha_{N}^{2}\left\{-\frac{\left[q(z) f(t) R_{1}\right]^{n}}{2(n+2)(n+3)} B R_{1}^{2}\left(\frac{R_{1}}{R}\right)\right. \\
\left.\quad \times\left[n(n+2)-n(n-1)(n+3)\left(\frac{k^{2}}{R_{1}}\right)-3\left(n^{2}+2 n-2\right)\left(\frac{k^{2}}{R_{1}}\right)^{n+3}\right]\right\} .
\end{aligned}
$$

From (2.20) and (3.27), (3.28), (3.29), (3.34), (3.35), and (3.36), the volume flow rate is calculated and is given by

$$
Q=4\left[\int_{0}^{R_{0 P}}\left(u_{0 P}+\alpha_{H}^{2} u_{1 P}\right) r d r+\int_{R_{0 P}}^{R_{1}}\left(u_{0 H}+\alpha_{H}^{2} u_{1 H}\right) r d r+\int_{R_{1}}^{R}\left(u_{0 N}+\alpha^{2} u_{1 N}\right) r d r\right]
$$


Mathematical Problems in Engineering

$$
\begin{aligned}
& =4[q(z) f(t) R] R^{3}\left\{1-\left(\frac{R_{1}}{R}\right)^{2}\right\}\left[\left(\frac{k^{2}}{R_{1}}\right)^{2}+\frac{1}{4}\left\{1-\left(\frac{R_{1}}{R}\right)^{2}\right\}\right] \\
& +\frac{4\left[q(z) f(t) R_{1}\right]^{n} R_{1}^{3}}{(n+2)(n+3)}\left[(n+2)-n(n+3)\left(\frac{k^{2}}{R_{1}}\right)+\left(n^{2}+2 n-2\right)\left(\frac{k^{2}}{R_{1}}\right)^{n+3}\right] \\
& +4 \alpha_{H}^{2}\left[-[q(z) f(t) R] B R^{2} R_{1}^{3}\left\{\frac{3}{32}\left(\frac{R}{R_{1}}\right)-\frac{1}{8}\left(\frac{R_{1}}{R}\right)+\frac{1}{32}\left(\frac{R_{1}}{R}\right)^{3}+\frac{1}{8}\left(\frac{R_{1}}{R}\right)^{3} \log \left(\frac{R_{1}}{R}\right)\right\}\right. \\
& +\left[q(z) f(t) R_{1}\right]^{n} B R_{1}^{5} \log \left(\frac{R_{1}}{R}\right) \\
& \times\left\{\frac{n}{2(n+3)}-\frac{n(n-1)}{2(n+2)}\left(\frac{k^{2}}{R_{1}}\right)-\frac{3\left(n^{2}+2 n-2\right)}{2(n+2)(n+3)}\left(\frac{k^{2}}{R_{1}}\right)^{n+3}\right\} \\
& -n\left[q(z) f(t) R_{1}\right]^{n} B R^{2} R_{1}^{3}\left\{1-\left(\frac{R_{1}}{R}\right)^{2}\right\} \\
& \times\left\{\frac{1}{4(n+3)}-\frac{(n-1)}{4(n+2)}\left(\frac{k^{2}}{R_{1}}\right)+\frac{\left(n^{2}+n-5\right)}{4(n+2)(n+3)}\left(\frac{k^{2}}{R_{1}}\right)^{n+3}\right\} \\
& -n\left[q(z) f(t) R_{1}\right]^{2 n-1} B R_{1}^{5} \\
& \times\left\{\frac{n}{2(n+2)(n+3)}-\frac{n(n-1)\left(4 n^{2}+12 n+5\right)}{(n+2)(n+3)(2 n+1)(2 n+3)}\left(\frac{k^{2}}{R_{1}}\right)\right. \\
& +\frac{n(n-1)^{2}}{2(n+1)(n+2)}\left(\frac{k^{2}}{R_{1}}\right)^{2}+\frac{\left(n^{3}-2 n^{2}-11 n+6\right)}{2(n+1)(n+2)(n+3)}\left(\frac{k^{2}}{R_{1}}\right)^{n+3} \\
& -\frac{(n-1)\left(n^{3}-2 n^{2}-11 n+6\right)}{2 n(n+2)(n+3)}\left(\frac{k^{2}}{R_{1}}\right)^{n+4} \\
& \left.\left.-\frac{\left(4 n^{5}+14 n^{4}-8 n^{3}-45 n^{2}-3 n+18\right)}{2 n(n+1)(n+2)(n+3)(2 n+3)}\left(\frac{k^{2}}{R_{1}}\right)^{2 n+4}\right\}\right] \\
& +4 \alpha_{N}^{2}\left[-[q(z) f(t) R] B R^{4} R_{1}\right. \\
& \times\left\{\frac{1}{24}\left(\frac{R}{R_{1}}\right)-\frac{3}{32}\left(\frac{R_{1}}{R}\right)+\frac{5}{96}\left(\frac{R_{1}}{R}\right)^{5}-\frac{1}{8}\left(\frac{R_{1}}{R}\right)^{3}\left(\log R_{1}\right)\left\{1-\left(\frac{R_{1}}{R}\right)^{2}\right\}\right\} \\
& -\left[q(z) f(t) R_{1}\right]^{n} B R^{2} R_{1}^{3}\left\{1-\left(\frac{R_{1}}{R}\right)^{2}\right\}\left(1+2 \log R_{1}\right) \\
& \left.\times\left\{\frac{n}{4(n+3)}-\frac{n(n-1)}{4(n+2)}\left(\frac{k^{2}}{R_{1}}\right)-\frac{3\left(n^{2}+2 n-2\right)}{4(n+2)(n+3)}\left(\frac{k^{2}}{R_{1}}\right)^{n+3}\right\}\right] .
\end{aligned}
$$


The second approximation to plug core radius $R_{1 P}$ can be obtained by neglecting the terms with $\alpha_{H}^{4}$ and higher powers of $\alpha_{H}$ in (3.7) in the following manner. The shear stress $\tau_{H}=$ $\tau_{0 H}+\alpha_{H}^{2} \tau_{1 H}$ at $r=R_{P}$ is given by

$$
\left|\tau_{0 H}+\alpha_{H}^{2} \tau_{1 H}\right|_{r=R_{P}}=\theta
$$

Equation (3.39) reflects the fact that on the boundary of the plug core region, the shear stress is the same as the yield stress. Using the Cityplace Taylor's series of $\tau_{0 H}$ and $\tau_{1 H}$ about $R_{0 P}$ and using $\left.\tau_{0 H}\right|_{r=R_{0 P}}=\theta$, we get

$$
R_{1 P}=\left[\frac{1}{q(z) f(t)}\right]\left[-\left.\tau_{1 H}\right|_{r=R_{0 P}}\right]
$$

With the help of (3.7), (3.30), (3.32), and (3.40), the expression for $R_{P}$ can be obtained as

$$
\begin{aligned}
R_{P}= & k^{2}+\left(\frac{B \alpha_{H}^{2} R^{2}}{4}\right)[q(z) f(t) R]\left(\frac{k^{2}}{R}\right)\left\{1-\left(\frac{R_{1}}{R}\right)^{2}\right\} \\
& +\frac{n B \alpha_{H}^{2} R_{1}^{2}}{2(n+1)}\left[q(z) f(t) R_{1}\right]^{n}\left\{\left(\frac{k^{2}}{R_{1}}\right)-\frac{\left(n^{2}-1\right)}{n}\left(\frac{k^{2}}{R_{1}}\right)^{2}-\left(\frac{k^{2}}{R_{1}}\right)^{n+2}\right\} .
\end{aligned}
$$

The resistance to flow in the artery is given by

$$
\Lambda=\frac{[q(z) f(t)]}{Q}
$$

When $R_{1}=R$, the present model reduces to the single fluid model (Herschel-Bulkley fluid model) and in such case, the expressions obtained in the present model for velocity $u_{H}$, shear stress $\tau_{H}$,wall shear stress $\tau_{w}$, flow rate $Q$, and plug core radius $R_{P}$ are in good agreement with those of Sankar and Hemalatha [2].

\section{Numerical Simulation of Results and Discussion}

The objective of the present model is to understand and bring out the salient features of the effects of the pulsatility of the flow, nonNewtonian nature of blood, peripheral layer and stenosis size on various flow quantities. It is generally observed that the typical value of the power law index $n$ for blood flow models is taken to lie between 0.9 and 1.1 and we have used the typical value of $n$ to be 0.95 for $n<1$ and 1.05 for $n>1$ [2]. Since the value of yield stress is 0.04 dyne $/ \mathrm{cm}^{2}$ for blood at a haematocrit of 40 [35], the nonNewtonian effects are more pronounced as the yield stress value increases, in particular, when it flows through narrow blood vessels. In diseased state, the value of yield stress is quite high (almost five times) [28]. In this study, we have used the range from 0.1 to 0.3 for the nondimensional yield stress $\theta$. To compare the present results with the earlier results, we have used the yield stress value as 
0.01 and 0.04 . Though the range of the amplitude $A$ varies from 0 to 1 , we use the range from 0.1 to 0.5 to pronounce its effect.

The ratio $\alpha\left(=\alpha_{N} / \alpha_{H}\right)$ between the pulsatile Reynolds numbers of the Newtonian fluid and Herschel-Bulkley fluid is called pulsatile Reynolds number ratio. Though the pulsatile Reynolds number ratio $\alpha$ ranges from 0 to 1 ; it is appropriate to assume its value as 0.5 [25]. Although the pulsatile Reynolds number $\alpha_{H}$ of the Herschel-Bulkley fluid also ranges from 0 to 1 [2], the values 0.5 and 0.25 are used to analyze its effect on the flow quantities. Given the values of $\alpha$ and $\alpha_{H}$, the value of $\alpha_{N}$ can be obtained from $\alpha=\alpha_{N} / \alpha_{H}$. The value of the ratio $\beta$ of central core radius $\beta \bar{R}_{0}$ to the normal artery radius $\bar{R}_{0}$ in the unobstructed artery is generally taken as 0.95 and 0.985 [25]. Following Shukla et al. [26], we have used the relations $R_{1}=\beta R$ and $\delta_{C}=\beta \delta_{P}$ to estimate $R_{1}$ and $\delta_{C}$. The maximum thickness of the stenosis in the peripheral region $\delta_{P}$ is taken in the range from 0.1 to 0.15 [25]. To compare the present results with the results of Sankar and Hemalatha [2] for single fluid model, we have used the value 0.2 for $\delta_{C}$. To deduce the present model to a single fluid model (Newtonian fluid model or Herschel-Bulkley fluid model) and to compare the results with earlier results, we have used the value of $\beta$ as 1 .

It is observed that in (3.38), $f(t), R$, and $\theta$ are known and $Q$ and $q(z)$ are the unknowns to be determined. A careful analysis of (3.38) reveals the fact that $q(z)$ is the pressure gradient of the steady flow. Thus, if steady flow is assumed, then (3.38) can be solved for $q(z)[2,10]$. For steady flow, (3.38) reduces to

$$
\begin{aligned}
& \left(R^{2}-R_{1}^{2}\right)\left[4 \theta^{2}\left(\frac{R}{R_{1}}\right)^{2}+\left(R^{2}-R_{1}^{2}\right)\right] x^{3}+\left[\frac{4}{(n+2)(n+3)}\right] \\
& \times\left\lfloor(n+2) R_{1}^{n+3} x^{n+3}-n(n+3) \theta R_{1}^{n+2} x^{n+2}+\left(n^{2}+2 n-2\right) \theta^{n+3}\right\rfloor-Q_{S} x^{3}=0
\end{aligned}
$$

where $x=q(z)$ and $Q_{S}$ is the steady state flow rate. Equation (4.1) can be solved for $x$ numerically for a given value of $n, Q_{S}$ and $\theta$. Equation (4.1) has been solved numerically for $x$ using Newton-Raphson method with variation in the axial direction and yield stress with $\beta=0.95$ and $\delta_{P}=0.1$. Throughout the analysis, the steady flow rate $Q_{S}$ value is taken as 1.0. Only that root which gives the realistic value for plug core radius has been considered (there are only two real roots in the range from 0 to 20 and the other root gives values of plug core radius that exceeds the tube radius $R$ ).

\subsection{Pressure Gradient}

The variation of pressure gradient with axial distance for different fluid models in the core region is shown in Figure 2. It has been observed that the pressure gradient for the Newtonian fluid (single fluid model) is lower than that of the two fluid models with $n=1.05$ and $\theta=$ 0.1 from $z=4$ to 4.5 and $z=5.5$ to 6 , and higher than that of the two fluid models from $z=4.5$ to $z=5.5$ and these ranges are changed with increase in the value of the yield stress $\theta$ and a decrease in the value of the power law index $n$. The plot for the Newtonian fluid model (single phase fluid model) is in good agreement with that in Figure 2 of Sankar and Hemalatha [2]. Figure 2 depicts the effects of nonNewtonian nature of blood on pressure gradient. 


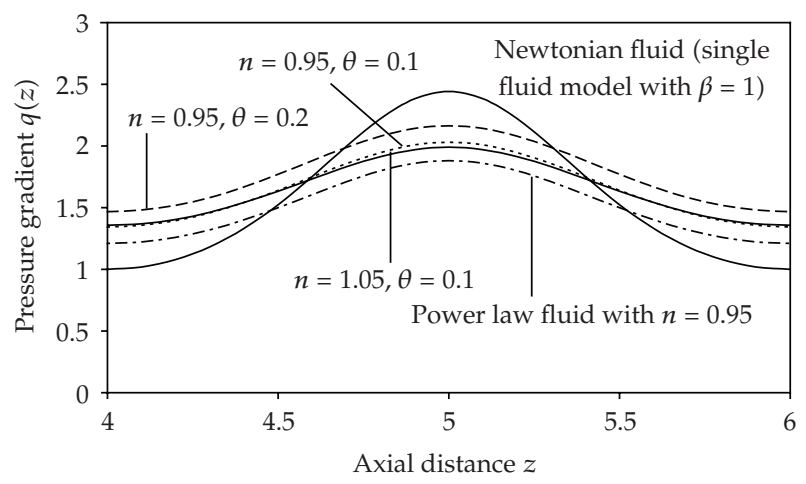

Figure 2: Variation of pressure gradient with axial direction for different fluids in the core region with $\delta_{P}=0.1$.

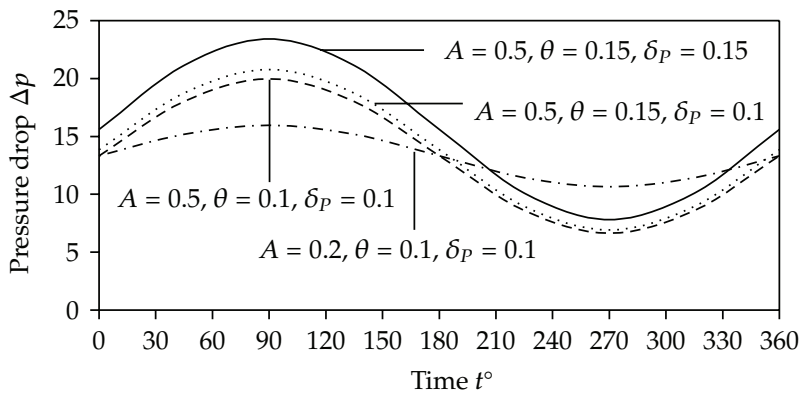

Figure 3: Variation of pressure drop in a time cycle for different values of $A, \theta$ and $\delta_{P}$ with $n=\beta=0.95$.

\subsection{Pressure Drop}

The variation of pressure drop $(\Delta p)$ (across the stenosis, i.e., from $z=4$ to $z=6$ ) in a time cycle for different values of $A, \theta$, and $\delta_{P}$ with $n=\beta=0.95$ is depicted in Figure 3. It is clear that the pressure drop increases as time $t$ increases from $0^{\circ}$ to $90^{\circ}$ and then decreases from $90^{\circ}$ to $270^{\circ}$ and again it increases from $270^{\circ}$ to $360^{\circ}$. The pressure drop is maximum at $90^{\circ}$ and minimum at $270^{\circ}$. It is also observed that for a given value of $A$, the pressure drop increases with the increase of the stenosis height $\delta_{P}$ or yield stress $\theta$ when the other parameters held constant. Further, it is noticed that as the amplitude $A$ increases, the pressure drop increases when $t$ lies between $0^{\circ}$ and $180^{\circ}$ and decreases when $t$ lies between $180^{\circ}$ and $360^{\circ}$ while $\theta$ and $\delta_{P}$ are held fixed. Figure 3 shows the simultaneous effects of the stenosis size and nonNewtonian nature of blood on pressure drop.

\subsection{Plug Core Radius}

The variation of plug core radius $\left(R_{P}\right)$ with axial distance for different values of the amplitude A and stenosis thickness $\delta_{P}$ (in the peripheral layer) with $n=\beta=0.95, \alpha_{H}=0.5, \theta=0.1$, and $t=60^{\circ}$ is shown in Figure 4. It is noted that the plug core radius decreases as the axial variable $z$ varies from 4 to 5 and it increases as $z$ varies from 5 to 6 . It is further observed that for a given value of $\delta_{P}$, the plug core radius decreases with the increase of the amplitude $A$ and the same behavior is noted as the peripheral layer stenosis thickness increases for a given value 


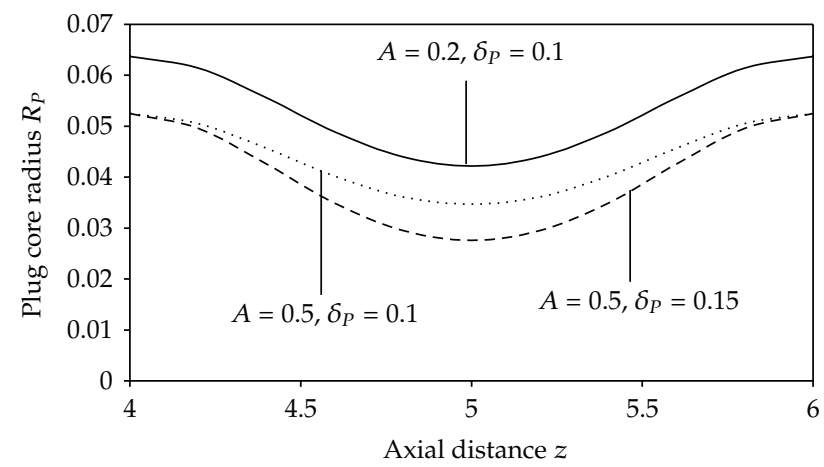

Figure 4: Variation of plug core radius with axial distance for different values of $A$ and $\delta_{P}$ with $n=\beta=0.95$, $\alpha_{H}=0.5, \theta=0.1$ and $t=60^{\circ}$.

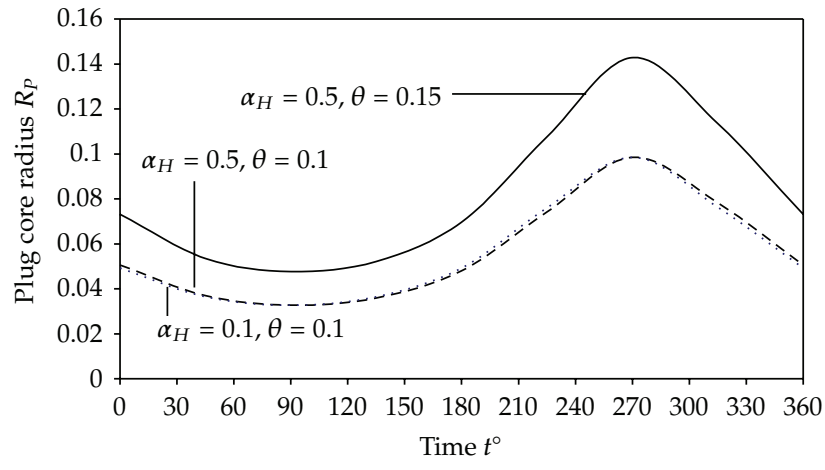

Figure 5: Variation of plug core radius in a time cycle for different values of $\alpha_{H}$ and $\theta$ with $n=\beta=0.95$, $\delta_{P}=0.1, A=0.5, t=60^{\circ}$ and $z=5$.

of the amplitude $A$. Figure 4 depicts the effects of stenosis height on the plug core radius of the blood vessels.

Figure 5 sketches the variation of plug core radius in a time cycle for different values of the pulsatile Reynolds number $\alpha_{H}$ of the Herschel-Bulkley fluid and yield stress $\theta$ with $n=\beta=0.95, A=0.5, z=5, t=60^{\circ}$, and $\delta_{P}=0.1$. It is noted that the plug core radius decreases as time $t$ increases from $0^{\circ}$ to $90^{\circ}$ and then it increases from $90^{\circ}$ to $270^{\circ}$ and then again it decreases from $270^{\circ}$ to $360^{\circ}$. The plug core radius is minimum at $t=90^{\circ}$ and maximum at $t=270^{\circ}$. It has been observed that for a given value of the pulsatile Reynolds number $\alpha_{H}$, the plug core radius increases as the yield stress $\theta$ increases. Also, it is noticed that for a given value of yield stress $\theta$ and with increasing values of the pulsatile Reynolds number $\alpha_{H}$, the plug core radius increases when $t$ lies between $0^{\circ}$ and $90^{\circ}$ and also between $270^{\circ}$ and $360^{\circ}$ and decreases when $t$ lies between $90^{\circ}$ and $270^{\circ}$. Figure 5 depicts the simultaneous effects of the pulsatility of the flow and the nonNewtonian nature of the blood on the plug core radius of the two-phase model.

\subsection{Wall Shear Stress}

Wall shear stress is an important parameter in the studies of the blood flow through arterial stenosis. Accurate predictions of wall shear stress distributions are particularly useful in the 


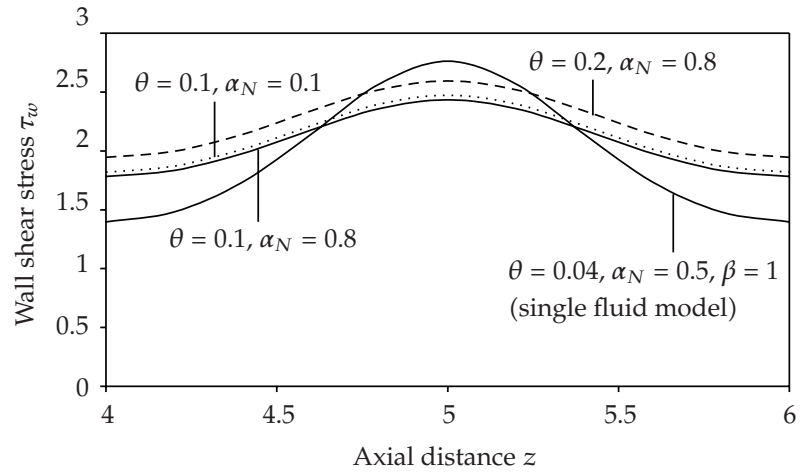

Figure 6: Variation of wall shear stress with axial distance for different values $\theta$ and $\alpha_{N}$ with $t=45^{\circ}$, $n=\beta=0.95, A=0.5$ and $\delta_{P}=0.1$.

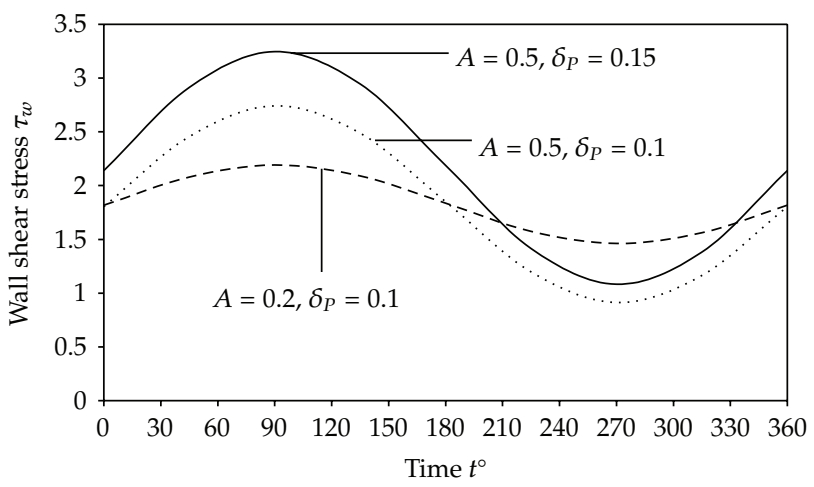

Figure 7: Variation of wall shear stress in a time cycle for different values of $A$ and $\delta_{P}$ with $\theta=0.1$, $n=\beta=0.95, z=5$ and $\alpha_{N}=0.5$.

understanding of the effects of blood flow on the endothelial cells [36, 37]. The variation of wall shear stress in the axial direction for different values of yield stress $\theta$ and pulsatile Reynolds number $\alpha_{N}$ of the Newtonian fluid with $t=45^{\circ}, n=\beta=0.95, A=0.5$, and $\delta_{P}=0.1$ is plotted in Figure 6. It is found that the wall shear stress increases as the axial variable $z$ increases from 4 to 5 and then it decreases symmetrically as $z$ increases further from 5 to 6 . For a given value of the pulsatile Reynolds number $\alpha_{N}$, the wall shear stress increases considerably with the increase in the values of the yield stress $\theta$ when the other parameters held constant. Also, it is noticed that for a given value of the yield stress $\theta$ and increasing values of the pulsatile Reynolds number $\alpha_{N}$, the wall shear stress decreases slightly while the other parameters are kept as invariables. It is of interest to note that the plot for the single fluid Herschel-Bulkley model is in good agreement with that in Figure 8 of Sankar and Hemalatha [2]. Figure 6 shows the effects of pulsatility of the blood flow and nonNewtonian effects of the blood on the wall shear stress of the two-phase model.

Figure 7 depicts the variation of wall shear stress in a time cycle for different values of the amplitude $A$ and peripheral stenosis height $\delta_{P}$ with $n=\beta=0.95, \theta=0.1, \alpha_{N}=0.5$ and $z=5$. It can be easily seen that the wall shear stress increases as time $t$ (in degrees) increases from $0^{\circ}$ to $90^{\circ}$ and then it decreases as $t$ increases from $90^{\circ}$ to $270^{\circ}$ and then again it increases as $t$ increases further from $270^{\circ}$ to $360^{\circ}$. The wall shear stress is maximum at $90^{\circ}$ and 


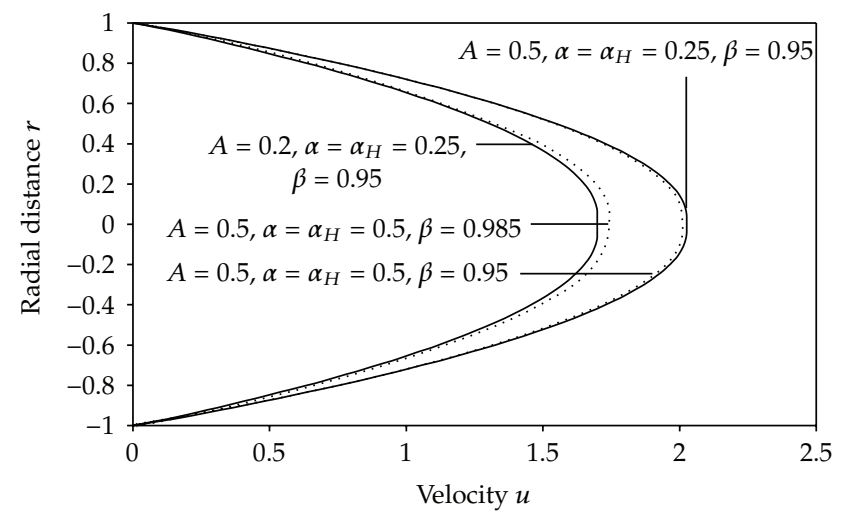

Figure 8: Velocity distribution for different values of $A, \alpha, \alpha_{H}$ and $\beta$ with $\theta=\delta_{P}=0.1, z=5, n=0.95$ and $t=45^{\circ}$.

minimum at $270^{\circ}$. Also, it may be noted that for a given value of the amplitude $A$ the wall shear stress increases with increasing values of the stenosis thickness $\delta_{P}$. Further, it is noticed that for a given value of the stenosis size and increasing values of the amplitude $A$, the wall shear stress increases when $t$ lies between $0^{\circ}$ and $180^{\circ}$ and decreases when $t$ lies between $180^{\circ}$ and $360^{\circ}$. This figure sketches the effects of the stenosis size and amplitude on the wall shear stress of the two-phase blood flow model.

\subsection{Velocity Distribution}

The velocity profiles are of interest, since they provide a detailed description of the flow field. The velocity distributions in the radial direction for different values of the amplitude $A$, pulsatile Reynolds number ratio $\alpha$, pulsatile Reynolds number of Herschel-Bulkley fluid $\alpha_{H}$, the ratio of the central core radius to the tube radius $\beta$ with $n=0.95, z=5, \theta=\delta_{P}=0.1$, and $t=45^{\circ}$ are shown in Figure 8. One can easily notice the plug flow around the tube axis in Figure 8. Also, it is found that the velocity increases as the amplitude $A$ increases for a given set of values of $\alpha, \alpha_{H}$ and $\beta$. Further, it is observed that for a given set of values of $A, \alpha$ and $\alpha_{H}$, the velocity decreases considerably near the tube axis as the ratio $\beta$ increases. The same behavior is observed for increasing values of the pulsatile Reynolds number ratio $\alpha$ and pulsatile Reynolds number $\alpha_{H}$ for the given values of $A$ and $\beta$, but there is only a slight decrease in the later case. Figure 8 depicts the effects of amplitude, pulsatility and stenosis size on velocity distribution of the two-phase model. The velocity distribution in the radial direction at different times is shown in Figure 9. It is observed that the velocity increases as time $t$ (in degrees) increases from $0^{\circ}$ to $90^{\circ}$ and then it decreases as $t$ increases from $90^{\circ}$ to $270^{\circ}$ and again it increases as $t$ increases further from $270^{\circ}$ to $360^{\circ}$. This figure shows the transient effects of blood flow on velocity of the two-phase model.

\subsection{Resistance to Flow}

The variation of resistance to flow with peripheral layer stenosis size for different values of the amplitude $A$ and yield stress $\theta$ with $n=\beta=0.95, \alpha=\alpha_{H}=0.25$, and $t=45^{\circ}$ is plotted in Figure 10. Since $\delta_{C}=\beta \delta_{P}$, the stenosis size of the core region $\delta_{C}$ also increases when the 


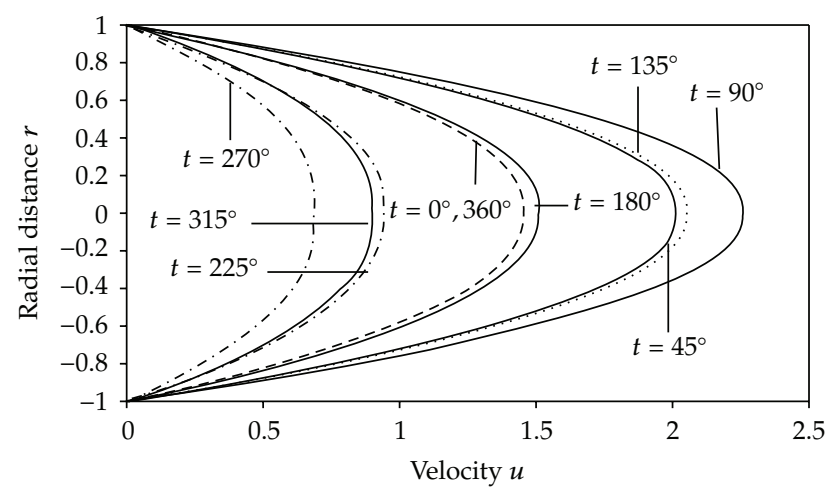

Figure 9: Velocity distribution at different times with $n=\beta=0.95, \theta=\delta_{P}=0.1, \alpha=\alpha_{H}=0.5, z=5$ and $A=0.5$.

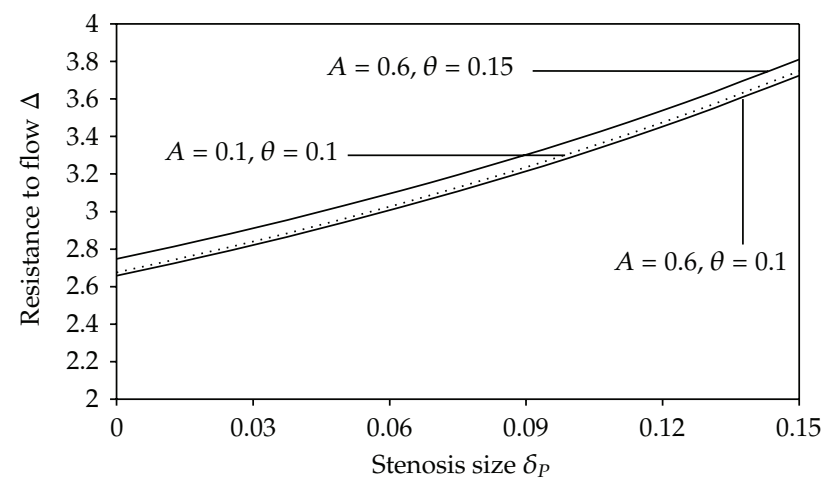

Figure 10: Variation of resistance to flow with stenosis size for different values of $A$ and $\theta$ with $n=\beta=0.95$, $\alpha=\alpha_{H}=0.25$ and $t=45^{\circ}$.

peripheral layer stenosis height $\delta_{P}$ increases for a given value of $\beta$. It is seen that the resistance to flow increases gradually with increasing stenosis size while the rest of the parameters are kept fixed. It is to be noted that for a given value of yield stress $\theta$, the resistance to flow decreases with increasing values of the amplitude $A$. It is also found that for a given value of the amplitude $A$, the resistance to flow increases with increase in the values of the yield stress $\theta$ when the other parameters held constant. Figure 10 illustrates the effects of the amplitude, stenosis size and the nonNewtonian nature of blood on resistance to flow of the two-phase model.

Figure 11 sketches the variation of resistance to flow in a time cycle for different values of the power law index $n$ and the pulsatile Reynolds number ratio $\alpha$, pulsatile Reynolds number of the Herschel-Bulkley fluid $\alpha_{H}$ with $\theta=\delta_{P}=0.1, \beta=0.95$ and $A=0.2$. It is clear that the resistance to flow decreases as time $t$ (in degrees) increases from $0^{\circ}$ to $90^{\circ}$ and then it increases as $t$ increases from $90^{\circ}$ to $270^{\circ}$ and then again it decreases as $t$ increases further from $270^{\circ}$ to $360^{\circ}$. The resistance to flow is minimum at $90^{\circ}$ and maximum at $270^{\circ}$. It is found that for the fixed values of $\alpha$ and $\alpha_{H}$ and the increasing values of the power law index $n$, the resistance to flow decreases when time $t$ lies between $0^{\circ}$ and $180^{\circ}$ and increases when $t$ lies between $180^{\circ}$ and $360^{\circ}$. Further, it is noted that for a fixed value of the power law index $n$ and with the increasing values of $\alpha$ and $\alpha_{H}$, the resistance to flow increases slightly when $t$ 


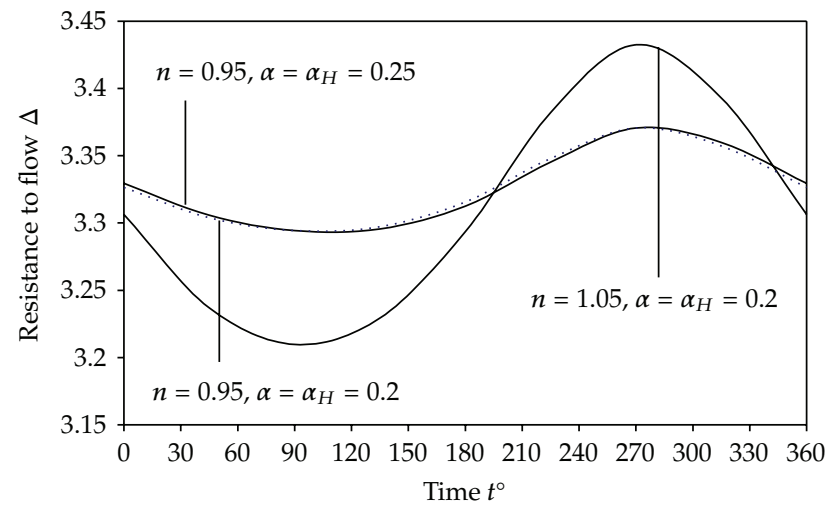

Figure 11: Variation of resistance to flow in a time cycle for different values of $\alpha, \alpha_{H}$ and $n$ with $\theta=\delta_{P}=0.1$, $\beta=0.95$, and $A=0.2$.

Table 1: Estimates of the wall shear stress increase factor for the two-phase Herschel-Bulkley fluid model and single-phase Herschel-Bulkley fluid model for different stenosis sizes with $n=0.95, A=\alpha=\alpha_{H}=0.5$, $\beta=0.985, \theta=0.1$, and $t=45^{\circ}$.

\begin{tabular}{lcc}
\hline Stenosis size $\left(\delta_{P}\right)$ & Two-phase fluid model & Single-phase fluid model \\
\hline 0.025 & 1.074 & 1.156 \\
0.05 & 1.157 & 1.350 \\
0.075 & 1.249 & 1.595 \\
0.1 & 1.352 & 1.907 \\
0.125 & 1.467 & 2.313 \\
0.15 & 1.597 & 2.848 \\
\hline
\end{tabular}

lies between $0^{\circ}$ and $90^{\circ}$ and also between $270^{\circ}$ and $360^{\circ}$ and decreases slightly when $t$ lies between $90^{\circ}$ and $270^{\circ}$. Figure 11 shows the simultaneous effects of pulsatility of the flow and the nonNewtonian nature of blood on resistance to flow of the two-phase model.

\subsection{Quantification of Wall Shear Stress and Resistance to Flow}

The wall shear stress $\left(\tau_{w}\right)$ and resistance to flow $(\Lambda)$ are physiologically important quantities which play an important role in the formation of platelets [38]. High wall shear stress not only damages the vessel wall and causes intimal thickening, but also activates platelets, cause platelet aggregation, and finally results in the formation of thrombus [7].

The wall shear stress increase factor is defined as the ratio of the wall shear stress of particular fluid model in the stenosed artery for a given set of values of the parameters to the wall shear stress of the same fluid model in the normal artery for the same set of values of the parameters. The estimates of the wall shear stress increase factor for twophase Herschel-Bulkley fluid model and single-phase fluid model with $t=45^{\circ}, n=0.95$, $A=\alpha=\alpha_{H}=0.5, \beta=0.985$, and $\theta=0.1$ are given in Table 1. It is observed that for the range of the stenosis size $0-0.15$, the corresponding ranges of the wall shear stress increase of the two-phase Herschel-Bulkley fluid model and single-phase Herschel-Bulkley fluid model are 1.074-1.594 and 1.156-2.848, respectively. It is found that the estimates of the wall shear 
Table 2: Estimates of the resistance to flow increase factor for the two-phase Herschel-Bulkley fluid model and single-phase Herschel-Bulkley fluid model for different stenosis sizes with $n=0.95, A=\alpha=\alpha_{H}=0.5$, $\beta=0.985, \theta=0.1$, and $t=45^{\circ}$.

\begin{tabular}{lcc}
\hline Stenosis size $\left(\delta_{P}\right)$ & Two-phase fluid model & Single-phase fluid model \\
\hline 0.025 & 1.050 & 1.104 \\
0.05 & 1.105 & 1.232 \\
0.075 & 1.166 & 1.391 \\
0.1 & 1.233 & 1.592 \\
0.125 & 1.308 & 1.850 \\
0.15 & 1.393 & 2.189 \\
\hline
\end{tabular}

stress increase factor are marginally lower for the two-phase Herschel-Bulkley fluid model than those of the single-phase Herschel-Bulkley fluid model.

One can define the resistance to flow increase factor in a similar way as in the case of wall shear stress increase factor. The estimates of the increase in resistance to flow factor for two-phase Herschel-Bulkley fluid model and single-phase fluid model with $t=45^{\circ}, n=$ 0.95, $A=\alpha=\alpha_{H}=0.5, \beta=0.985$, and $\theta=0.1$ are given in Table 2. It is noted that for the range of the stenosis size $0-0.15$, the corresponding range of the increase in resistance to flow factor for the two-phase Herschel-Bulkley fluid model and single-phase Herschel-Bulkley fluid model are 1.050-1.393 and 1.104-2.189, respectively. It is found that the estimates of the wall shear stress increase factor are significantly lower for the two-phase Herschel-Bulkley fluid model than those of the single-phase Herschel-Bulkley fluid model. Hence, it is clear that the existence of the peripheral layer is useful in the functioning of the diseased arterial system. It is strongly felt that the present model may provide a better insight to the study of blood flow behavior in the stenosed arteries than the earlier models.

Perturbation method is a very useful analytical tool for solving nonlinear differential equations. In the present study, it is used to solve the nonlinear coupled implicit system of partial differential equations to get an asymptotic solution. This method yields a closed form to the flow quantities which enables us to evaluate them at any particular instant of time and at any particular point in the flow domain. This facility is unavailable when we use the computational methods such as finite difference method, finite element method, finite volume method.

\section{Conclusion}

The present study analyzes the two-phase Herschel-Bulkley fluid model for blood flow through stenosed arteries and brings out many interesting fluid mechanical phenomena due to the presence of the peripheral layer. The results indicate that the pressure drop, plug core radius, wall shear stress, and resistance to flow increase as the yield stress or stenosis size increases while all other parameters held constant. It is found that the velocity increases, plug core radius, and resistance to flow decrease as the amplitude increases. It is also observed that the difference between the estimates of increase in the wall shear stress factor of the two-phase fluid model and single-phase fluid model is substantial. A similar behavior is observed for the increase in resistance to flow factor. Thus, the results demonstrate that this model is capable of predicting the hemodynamic features most interesting to physiologists. Thus, the present study could be useful for analyzing the blood flow in the diseased state. From this study, it is 
concluded that the presence of the peripheral layer (outer phase) helps in the functioning of the diseased arterial system.

\section{Nomenclature}

$\bar{r}$ : $\quad$ radial distance

$r$ : dimensionless radial distance

$\bar{z}: \quad$ axial distance

$z$ : dimensionless axial distance

$n: \quad$ power law index

$\bar{p}: \quad$ pressure

$p: \quad$ dimensionless pressure

$\bar{Q}: \quad$ flow rate

$Q: \quad$ dimensionless flow rate

$\bar{R}_{0}$ : $\quad$ radius of the normal artery

$\bar{R}(\bar{z})$ : radius of the artery in the stenosed peripheral region

$R(z)$ : dimensionless radius of the artery in the stenosed peripheral region

$\bar{R}_{1}(\bar{z})$ : radius of the artery in the stenosed core region

$R_{1}(z)$ : dimensionless radius of the artery in the stenosed core region

$\bar{R}_{P}: \quad$ plug core radius

$R_{P}$ : dimensionless plug core radius

$\bar{u}_{H}: \quad$ axial velocity of the Herschel-Bulkley fluid

$u_{H}$ : dimensionless axial velocity of the Herschel-Bulkley fluid

$\bar{u}_{N}: \quad$ axial velocity of the Newtonian fluid

$u_{N}$ : dimensionless axial velocity of the Newtonian fluid

A: amplitude of the flow

$\bar{q}(\bar{z}):$ steady state pressure gradient

$q(z)$ : dimensionless steady state pressure gradient

$\bar{q}_{0}: \quad$ negative of the pressure gradient in the normal artery

$\bar{L}$ : length of the normal artery

$\bar{L}_{0}$ : length of the stenosis

$L_{0}$ : dimensionless length of the stenosis

$\bar{d}$ : location of the stenosis

$d$ : dimensionless location of the stenosis

$\bar{t}: \quad$ time

$t$ : dimensionless time.

\section{Greek Letters}

$\Delta p$ : dimensionless Pressure drop

$\Lambda$ : dimensionless resistance to flow

$\phi$ : azimuthal angle

$\dot{\gamma}$ : shear rate

$\bar{\tau}_{y}:$ yield stress

$\theta:$ dimensionless yield stress

$\bar{\tau}_{H}$ : shear stress for the Herschel-Bulkley fluid

$\tau_{H}$ : dimensionless shear stress for the Herschel-Bulkley fluid 
$\bar{\tau}_{N}$ : shear stress for the Newtonian fluid

$\tau_{N}$ : dimensionless shear stress for the Newtonian fluid

$\tau_{w}$ : dimensionless wall shear stress

$\bar{\rho}_{H}$ : density of the Herschel-Bulkley fluid

$\bar{\rho}_{N}$ : density of the Newtonian fluid

$\bar{\mu}_{H}$ : viscosity of the Herschel-Bulkley fluid

$\bar{\mu}_{N}$ : viscosity of the Newtonian fluid

$\alpha_{H}$ : pulsatile Reynolds number of the Herschel-Bulkley fluid

$\alpha_{N}$ : pulsatile Reynolds number of the Newtonian fluid

$\alpha$ : ratio between the Reynolds numbers $\alpha_{H}$ and $\alpha_{N}$

$\beta$ : ratio of the central core radius to the normal artery radius

$\bar{\delta}_{C}:$ maximum height of the stenosis in the core region

$\delta_{C}$ : dimensionless maximum height of the stenosis in the core region

$\bar{\delta}_{N}$ : maximum height of the stenosis in the peripheral region

$\delta_{P}$ : dimensionless maximum height of the stenosis in the peripheral region

$\bar{\omega}$ : angular frequency of the blood flow.

\section{Subscripts}

$w$ : wall shear stress (used for $\tau$ )

$C$ : core region (used for $\bar{\delta}, \delta$ )

$P$ : peripheral region (used for $\bar{\delta}, \delta$ )

$H$ : herschel-Bulkley fluid (used for $\bar{u}, u, \bar{\tau}, \tau$ )

$N$ : newtonian fluid (used for $\bar{u}, u, \bar{\tau}, \tau$ ).

\section{Acknowledgment}

The present work is financially supported by the research university grant of Universiti Sains Malaysia, Malaysia (Grant Ref. No: 1001/PMATHS/816088).

\section{References}

[1] D. Liepsch, M. Singh, and M. Lee, "Experimental analysis of the influence of stenotic geometry on steady flow," Biorheology, vol. 29, no. 4, pp. 419-431, 1992.

[2] D. S. Sankar and K. Hemalatha, "Pulsatile flow of Herschel-Bulkley fluid through stenosed arteries-A mathematical model," International Journal of Non-Linear Mechanics, vol. 41, no. 8, pp. 979-990, 2006.

[3] M. S. Moayeri and G. R. Zendehbudi, "Effects of elastic property of the wall on flow characteristics through arterial stenoses," Journal of Biomechanics, vol. 36, no. 4, pp. 525-535, 2003.

[4] P. K. Mandal, "An unsteady analysis of non-Newtonian blood flow through tapered arteries with a stenosis," International Journal of Non-Linear Mechanics, vol. 40, no. 1, pp. 151-164, 2005.

[5] I. Marshall, S. Zhao, P. Papathanasopoulou, P. Hoskins, and X. Y. Xu, "MRI and CFD studies of pulsatile flow in healthy and stenosed carotid bifurcation models," Journal of Biomechanics, vol. 37, no. 5, pp. 679-687, 2004.

[6] S. Chakravarty and P. K. Mandal, "Two-dimensional blood flow through tapered arteries under stenotic conditions," International Journal of Non-Linear Mechanics, vol. 35, no. 5, pp. 779-793, 2000.

[7] G.-T. Liu, X.-J. Wang, B.-Q. Ai, and L.-G. Liu, "Numerical study of pulsating flow through a tapered artery with stenosis," Chinese Journal of Physics, vol. 42, no. 4, pp. 401-409, 2004.

[8] Q. Long, X. Y. Xu, K. V. Ramnarine, and P. Hoskins, "Numerical investigation of physiologically realistic pulsatile flow through arterial stenosis," Journal of Biomechanics, vol. 34, no. 10, pp. 1229-1242, 2001. 
[9] C. Tu and M. Deville, "Pulsatile flow of Non-Newtonian fluids through arterial stenoses," Journal of Biomechanics, vol. 29, no. 7, pp. 899-908, 1996.

[10] P. Chaturani and R. P. Samy, "Pulsatile flow of Casson's fluid through stenosed arteries with applications to blood flow," Biorheology, vol. 23, no. 5, pp. 499-511, 1986.

[11] M. Texon, "A hemodynamic concept of atherosclerosis with particular reference to coronary occlusion," Archives of Internal Medicine, vol. 99, pp. 418-430, 1957.

[12] M. Texon, "The hemodynamic concept of atherosclerosis," Bulletin of the New York Academy of Medicine, vol. 36, pp. 263-273, 1960.

[13] D. F. Young and F. Y. Tsai, "Flow characteristics in models of arterial stenoses: I. Steady flow," Journal of Biomechanics, vol. 6, no. 4, pp. 395-410, 1973.

[14] B. E. Morgan and D. F. Young, "An integral method for the analysis of flow in arterial stenoses," Bulletin of Mathematical Biology, vol. 36, no. 1, pp. 39-53, 1974.

[15] D. A. MacDonald, “On steady flow through modeled vascular stenosis," Journal of Biomechanics, vol. 12, pp. 13-20, 1979.

[16] D. F. Young, "Fluid mechanics of arterial stenosis," Journal of Biomechanical Engineering, vol. 101, no. 3, pp. 157-175, 1979.

[17] A. Sarkar and G. Jayaraman, "Correction to flow rate-pressure drop relation in coronary angioplasty: steady streaming effect," Journal of Biomechanics, vol. 31, no. 9, pp. 781-791, 1998.

[18] R. K. Dash, G. Jayaraman, and K. N. Mehta, "Flow in a catheterized curved artery with stenosis," Journal of Biomechanics, vol. 32, no. 1, pp. 49-61, 1999.

[19] S. Chakravarty, A. Datta, and P. K. Mandal, "Analysis of nonlinear blood flow in a stenosed flexible artery," International Journal of Engineering Science, vol. 33, no. 12, pp. 1821-1837, 1995.

[20] S. Charm and G. Kurland, "Viscometry of human blood for shear rates of 0-100,000 sec ${ }^{-1}$," Nature, vol. 206, no. 4984, pp. 617-618, 1965.

[21] C. D. Han and B. Barnett, Measurement of Rheological Properties of Biological Fluids, edited by H. L. Gabelnick, M. Litt, Charles C. Thomas, Springfield, Ill, USA, 1973.

[22] C. E. Huckabe and A. W. Hahn, "A generalized approach to the modeling of arterial blood flow," The Bulletin of Mathematical Biophysics, vol. 30, no. 4, pp. 645-662, 1968.

[23] E. W. Merrill, "Rheology of human blood and some speculations on its role in vascular homeostasis," in Biomechanical Mechanisms in Vascular Homeostasis and Intravascular Thrombus, P. N. Sawyer, Ed., Appleton Century Crafts, New York, NY, USA, 1965.

[24] R. L. Whitemore, Rheology of the Circulation, Pergamon Press, New York, NY, USA, 1968.

[25] V. P. Srivastava and M. Saxena, "Two-layered model of casson fluid flow through stenotic blood vessels: applications to the cardiovascular system," Journal of Biomechanics, vol. 27, no. 7, pp. 921-928, 1994.

[26] J. B. Shukla, R. S. Parihar, and S. P. Gupta, "Effects of peripheral layer viscosity on blood flow through the artery with mild stenosis," Bulletin of Mathematical Biology, vol. 42, no. 6, pp. 797-805, 1980.

[27] J. B. Shukla, R. S. Parihar, and B. R. P. Rao, "Effects of stenosis on non-Newtonian flow of the blood in an artery," Bulletin of Mathematical Biology, vol. 42, no. 3, pp. 283-294, 1980.

[28] P. Chaturani and V. R. Ponnalagar Samy, "A study of non-Newtonian aspects of blood flow through stenosed arteries and its applications in arterial diseases," Biorheology, vol. 22, no. 6, pp. 521-531, 1985.

[29] G. Bugliarello and J. Sevilla, "Velocity distribution and other characteristics of steady and pulsatile blood flow in fine glass tubes," Biorheology, vol. 7, no. 2, pp. 85-107, 1970.

[30] G. R. Cokelet, "The rheology of human blood," in Biomechanics, Y. C. Fung, Ed., pp. 63-103, PrenticeHall, Englewood Cliffs, NJ, USA, 1972.

[31] V. P. Srivastava, "Arterial blood flow through a nonsymmetrical stenosis with applications," Japanese Journal of Applied Physics, vol. 34, no. 12, pp. 6539-6545, 1995.

[32] V. P. Srivastava, "Two-phase model of blood flow through stenosed tubes in the presence of a peripheral layer: applications," Journal of Biomechanics, vol. 29, no. 10, pp. 1377-1382, 1996.

[33] R. N. Pralhad and D. H. Schultz, "Two-layered blood flow in stenosed tubes for different diseases," Biorheology, vol. 25, no. 5, pp. 715-726, 1988.

[34] N. Iida, "Influence of plasma layer on steady blood flow in micro vessels," Japanese Journal of Applied Physics, vol. 17, pp. 203-214, 1978. 
[35] E. W. Errill, “Rheology of blood," Physiological Reviews, vol. 49, no. 4, pp. 863-888, 1969.

[36] J.-J. Chiu, D. L. Wang, S. Chien, R. Skalak, and S. Usami, "Effects of disturbed flow on endothelial cells," Journal of Biomechanical Engineering, vol. 120, no. 1, pp. 2-8, 1998.

[37] G. G. Galbraith, R. Skalak, and S. Chien, "Shear stress induces spatial reorganization of the endothelial cell cytoskeleton," Cell Motility and the Cytoskeleton, vol. 40, no. 4, pp. 317-330, 1998.

[38] T. Karino and H. L. Goldsmith, "Flow behaviour of blood cells and rigid spheres in an annular vortex," Philosophical Transactions of the Royal Society of London B, vol. 279, no. 967, pp. 413-445, 1977. 


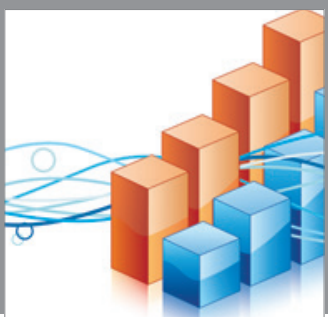

Advances in

Operations Research

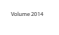

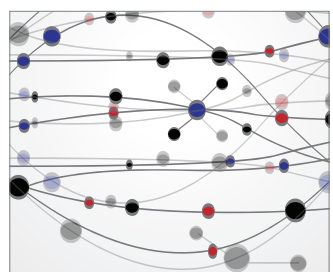

\section{The Scientific} World Journal
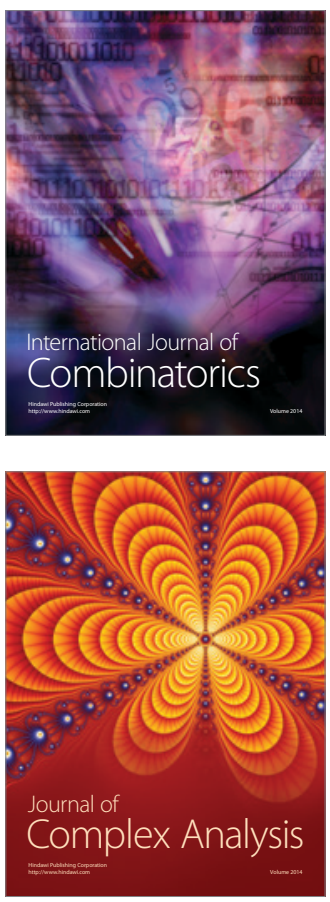

International Journal of

Mathematics and

Mathematical

Sciences
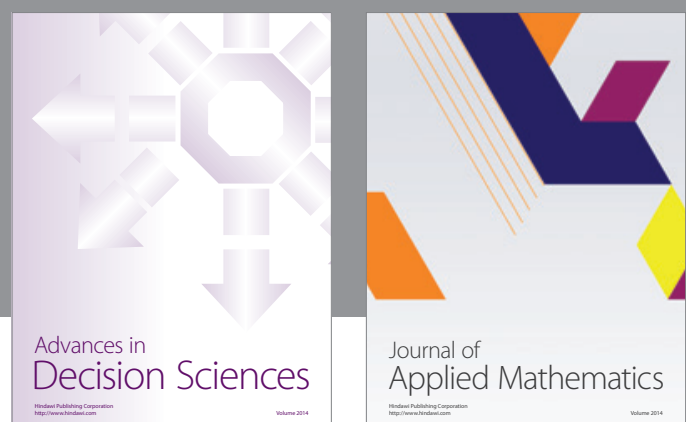

Journal of

Applied Mathematics
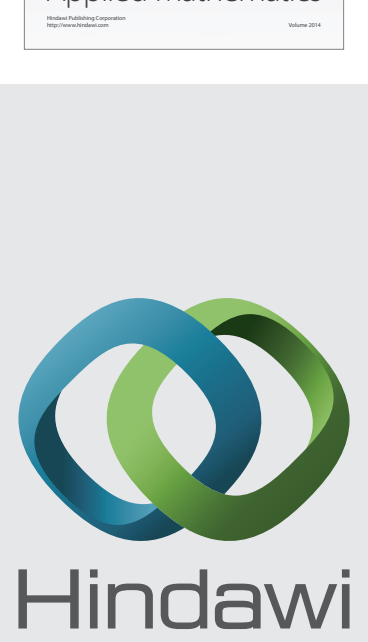

Submit your manuscripts at http://www.hindawi.com
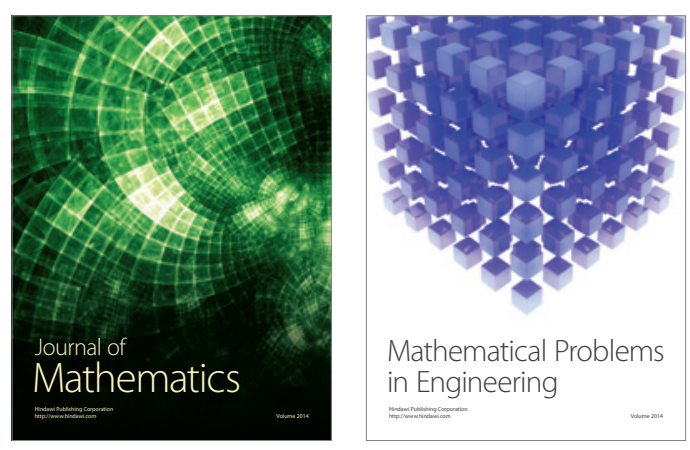

Mathematical Problems in Engineering
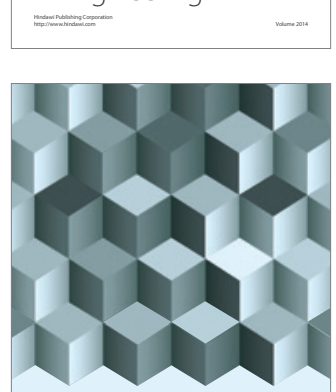

Journal of

Function Spaces
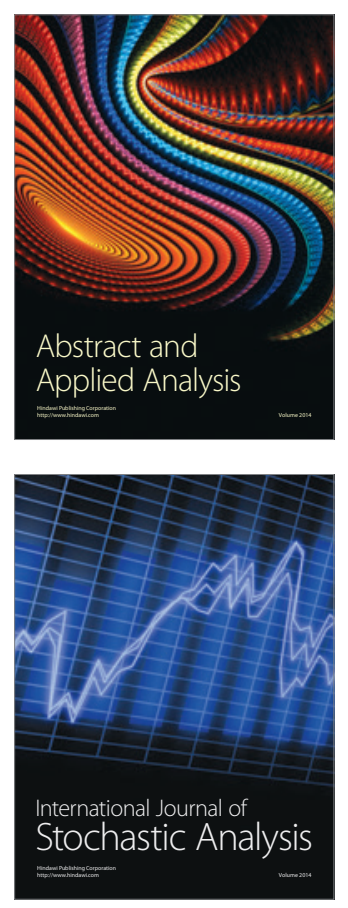

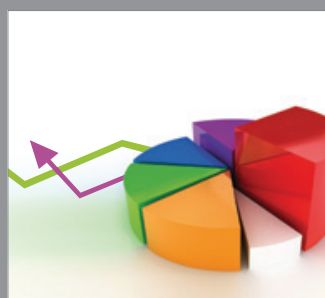

ournal of

Probability and Statistics

Promensencen
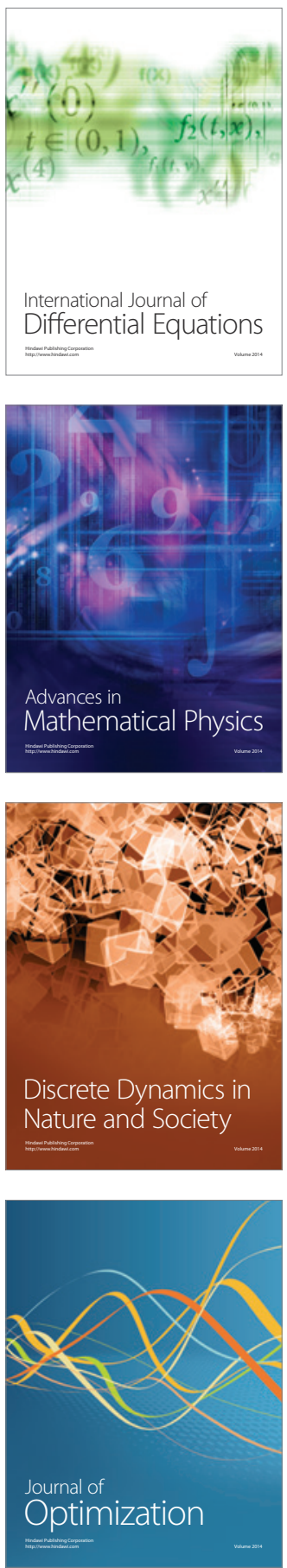